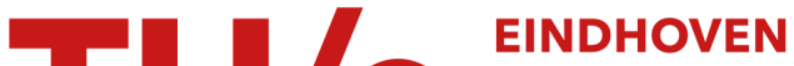 \\ UNIVERSITY OF \\ TECHNOLOGY
}

\section{Control to facet by piecewise-affine output feedback}

Citation for published version (APA):

Habets, L. C. G. J. M., Collins, P. J., \& Schuppen, van, J. H. (2012). Control to facet by piecewise-affine output feedback. IEEE Transactions on Automatic Control, 57(11), 2831-2843.

https://doi.org/10.1109/TAC.2012.2196401

DOI:

10.1109/TAC.2012.2196401

Document status and date:

Published: 01/01/2012

\section{Document Version:}

Publisher's PDF, also known as Version of Record (includes final page, issue and volume numbers)

\section{Please check the document version of this publication:}

- A submitted manuscript is the version of the article upon submission and before peer-review. There can be important differences between the submitted version and the official published version of record. People interested in the research are advised to contact the author for the final version of the publication, or visit the $\mathrm{DOI}$ to the publisher's website.

- The final author version and the galley proof are versions of the publication after peer review.

- The final published version features the final layout of the paper including the volume, issue and page numbers.

Link to publication

\section{General rights}

Copyright and moral rights for the publications made accessible in the public portal are retained by the authors and/or other copyright owners and it is a condition of accessing publications that users recognise and abide by the legal requirements associated with these rights.

- Users may download and print one copy of any publication from the public portal for the purpose of private study or research.

- You may not further distribute the material or use it for any profit-making activity or commercial gain

- You may freely distribute the URL identifying the publication in the public portal.

If the publication is distributed under the terms of Article 25fa of the Dutch Copyright Act, indicated by the "Taverne" license above, please follow below link for the End User Agreement:

www.tue.nl/taverne

Take down policy

If you believe that this document breaches copyright please contact us at:

openaccess@tue.nl

providing details and we will investigate your claim. 


\title{
Control to Facet by Piecewise-Affine Output Feedback
}

\author{
Luc C. G. J. M. Habets, Pieter J. Collins, and Jan H. van Schuppen
}

\begin{abstract}
The control-to-facet problem plays an important role in the design of feedback controllers for piecewise-affine hybrid systems on polytopes. In the literature, necessary conditions and sufficient conditions for solvability by static state feedback exist. In this paper, we extend these results to the case of continuous piecewise-affine static output feedback. For the construction of a controller, a triangulation of the output polytope is made which satisfies additional conditions to guarantee compatibility with the induced subdivision of the state polytope. In the state feedback case, the use of this special type of triangulation is not required.
\end{abstract}

Index Terms-Control system synthesis, output feedback, piecewise-affine hybrid systems, triangulation of polytopes.

\section{INTRODUCTION AND MOTIVATION}

I $\mathrm{N}$ the past 15 years, the study of hybrid systems has become a very active research area. Reasons for this rapidly growing interest are manifold. For example, if an engineering system, described by continuous-time dynamics, is controlled by a computer, then the closed-loop system is hybrid in the sense that its behavior is determined by the interaction between the continuous dynamics of a physical system, and the discrete dynamics that model the operation of a computer. Furthermore, the dynamics of control systems often contain discontinuities, or become hybrid after modeling. Examples of hybrid systems are abundantly available, ranging from the modeling of car engines to (air) traffic control and robot motion planning.

One particular class of hybrid systems, the class of piecewise-affine hybrid systems, has received a large amount of attention. A piecewise-affine hybrid system consists of a discrete automaton, with a continuous-time affine system on a polyhedral set at each discrete mode, and a switching mechanism between discrete and continuous dynamics. This class of systems was introduced by Sontag in [20] and [21], and has become popular because it is appropriate for the modeling of many real-world

Manuscript received June 30, 2010; revised July 21, 2011; accepted April 17, 2012. Date of publication April 25, 2012; date of current version October 24, 2012. This work was supported in part by the European Community's Seventh Framework Programme (FP7/2007-2013) under Grant Agreement INFSO-ICT223844. P. Collins was supported by the Nederlandse Organisatie voor Wetenschappelijk Onderzoek (NWO) Vidi grant 639.032.408. Recommended by Associate Editor Y. Hong.

L. C. G. J. M. Habets is with the Department of Mathematics and Computer Science, Technische Universiteit Eindhoven, NL-5600 MB Eindhoven, The Netherlands, and also with CWI, Amsterdam, The Netherlands (e-mail: 1.c.g.j.m.habets@tue.nl; luc.habets@cwi.nl).

P. J. Collins is with the Department of Knowledge Engineering, Maastricht University, NL-6200 MD Maastricht, The Netherlands (e-mail: pieter.collins@maastrichtuniversity.nl).

J. H. van Schuppen is with Centrum Wiskunde \& Informatica (CWI), NL-1090 GB Amsterdam, The Netherlands (e-mail: j.h.van.schuppen@cwi.nl).

Digital Object Identifier 10.1109/TAC.2012.2196401 systems (see, e.g., [1] and [7]), and its mathematical structure allows for useful theoretical results.

Several approaches exist for controlling piecewise-affine hybrid systems. A popular approach, developed by Morari and Bemporad et al. (see, e.g., [3] and [4]) is based on time discretization of the system, and is geared to performance optimization by computational methods. A completely different methodology is the so-called control-to-facet approach (see [9]-[11]). The idea of this approach is to apply continuous state feedback at each discrete mode of the hybrid system in order to influence the switching behavior of the underlying discrete automaton. Assuming that the facet through which the continuous state leaves the state polytope determines the next transition of the underlying discrete automaton, a central question in this method is how an affine system on a polytope can be steered to a particular (set of) facet(s), without leaving the state polytope before reaching this so-called exit facet.

The solution to the control-to-facet problem is an important building block for the control of hybrid systems. The problem of facet-blocking can be solved by linear programming and, hence, has polynomial complexity, though the full control-tofacet problem is NP-complete due to the need to solve a bi-linear inequality to force exit [18]. The method has been implemented in [6] and [8]. In [11], the control-to-facet method has been combined with a backward recursion algorithm on the discrete dynamics in order to achieve some a priori given control objectives. A related method for the control of multi-affine systems on rectangles is developed in [2].

Whereas in [9]-[11] the facet-blocking and control-to-facet problem were studied from a rather general point of view, some special cases were investigated in more detail by Broucke and co-workers. In [19], [13], and [14], the class of so-called affine hypersurface systems, i.e., affine systems on $n$-dimensional polytopes, with $n-1$ independent inputs, was studied, both on simplices [19], [13] and on general polytopes [14]. In the simplex case, a necessary and sufficient condition to solve the control-to-facet problem using continuous state feedback was obtained, and it was shown that this feedback is realizable by affine state feedback. On general polytopes, similar results were obtained under a rather mild controllability condition, and with affine feedback replaced by piecewise-affine feedback. In [5], the equivalence of continuous and affine state feedback for solving the control-to-facet problem was studied for affine systems on simplices that are not necessarily hypersurface systems.

Also in this paper we study the problems of facet blocking and control-to-facet for affine systems on polytopes, but instead of using state feedback, like in the references mentioned above, we attempt to solve these problems by static output feedback. 
So we assume that only partial observations are available, and that instead of the complete state, only the output may be used for feedback. The output feedback is assumed to be static in the sense that the input is a function of the output at the same time instant.

In [9] and [11], solvability conditions for the state feedback case are described by linear inequalities at the vertices of the state polytope, and a continuous piecewise-affine state feedback can be constructed after triangulation of the state polytope. The problem in the static output feedback case is that the observed output is the same on an affine subspace of states which may intersect more than one facet. For a given observed output, the input has to be chosen in such a way that it is suitable for all states that correspond to this output simultaneously. In particular, the input has to satisfy several linear inequalities at the same time. To handle this more complicated situation, we first construct a special subdivision of the output polytope based on a notion of compatibility with the state polytope and the output map. For this purpose the so-called chamber complex [16], [17] is used. In this setting, one necessary condition and one sufficient condition for solvability of the control-to-facet problem can be obtained in terms of linear inequalities at the vertices of the chamber complex. Next, a triangulation of the output polytope is made that is a refinement of the chamber complex. Based on this triangulation and the computed inputs at its vertices, a continuous piecewise-affine static output feedback is constructed that solves the control-to-facet problem.

The main differences between the state feedback solution to the control-to-facet problem described in [9] and [11], and the static output feedback solution that is presented in this paper, are as follows.

1) In the state feedback case, the solvability conditions are stated on the inputs at the vertices of the state polytope. For static output feedback it is not enough to consider only the inputs at the vertices of the output polytope; in Section IV it will be shown that all vertices of the chamber complex of the output polytope have to be checked.

2) In the state feedback case described in [9] and [11], any triangulation of the state polytope can be used for the construction of a piecewise-affine feedback law. For static output feedback, only so-called compatible triangulations can be applied. In particular, the triangulation of the output polytope has to be a refinement of the chamber complex.

The case of dynamic output feedback has also been considered by the authors, and can be reduced to the case of static output feedback. The method relies on constructing an observer with sufficiently high gain such that the error in estimating the observable part of the system state by the observer state is negligible. The details of this reduction are beyond the scope of this paper, and will be presented elsewhere.

This paper is organized as follows. Section II contains the exact problem formulation, and Section III is devoted to compatibility of subdivisions and to triangulation of the output polytope. A solution for the static output feedback case is presented in Section IV. In Section V, some examples are given, and we end with some concluding remarks in Section VI. In Appendix A, we present complete statements and proofs of the results on convex polytopes that are needed throughout the paper.

\section{Problem Description}

Let $X \subset \mathbb{R}^{n}$ be a full-dimensional polytope, and denote by $\mathcal{V}(X)$ the set of vertices of $X$, and by $\mathcal{F}(X)$ the set of all faces of $X$. More specifically, for integers $0 \leq k \leq n$, we denote by $\mathcal{F}_{k}(X)$ the set of all faces of $X$ of dimension $k$. In particular, $\mathcal{F}_{n-1}(X)$ is the set of all facets of $X$, and $\mathcal{F}_{0}(X)$ is the set of all vertices. Furthermore, if $\hat{x}$ is a point on the boundary of $X$, then $\mathcal{F}_{n-1}(\hat{x}, X)$ denotes the set of all facets of $X$ that contain the point $\hat{x}$.

Let $f: \mathbb{R}^{n} \rightarrow \mathbb{R}^{n}$ be a Lipschitz-continuous function, and consider the autonomous system $\dot{x}=f(x)$ on the polytope $X$. Let $F$ be a facet of $X$, with normal vector $n_{F}$, pointing out of $X$. Then the exit set $\operatorname{Ex}(F)$ of $F$ is defined as the set of points of $F$ through which a state trajectory $x(t)$ can leave $X$, i.e.,

$$
\operatorname{Ex}(F)=\operatorname{cl}\left\{x \in F \mid n_{F}^{T} f(x)>0\right\} .
$$

Facet $F$ is blocked if $\operatorname{Ex}(F)=\varnothing$, i.e., $n_{F}^{T} f(x) \leq 0$ for all $x \in F$.

Definition II.1: Let $x\left(t, x_{0}\right)$ be the trajectory of the autonomous system $\dot{x}=f(x)$ on polytope $X$, with initial state $x_{0} \in X$. This trajectory is said to leave polytope $X$ by crossing facet $F$ of $X$ at time $T \geq 0$ if:

(i) $\forall t \in[0, T]: x\left(t, x_{0}\right) \in X$;

(ii) $x\left(T, x_{0}\right)$ is an element of the exit set of $F$;

(iii) $\exists \varepsilon>0, \forall t \in(T, T+\varepsilon): x\left(t, x_{0}\right) \notin X$.

Note that in condition (iii), we consider an extension of the trajectory outside the polytope. If $f$ is only defined on $X$ itself, then $f$ may be temporarily extended to a Lipschitz-continuous function on $\mathbb{R}^{n}$. This extension is only required for a correct interpretation of condition (iii) of Definition II.1, and is not used elsewhere in this paper. It is straightforward to show that condition (iii) is independent of the extension that is chosen.

Remark II.2: Definition II.1, adopted from [11, Def. 4.5], is a slight modification of the control-to-facet problem as formulated in, e.g., [10], [5], [18], [19], and [13]. In these papers, condition (ii) states that $x\left(T, x_{0}\right)$ may be any point of the facet $F$. In Definition II.1, condition (ii) is slightly more restrictive, because it requires that $x\left(T, x_{0}\right)$ belongs to the exit set of facet $F$. The reason for defining crossing a facet in this way is the applicability of our results in the framework of piecewise-affine hybrid systems on polytopes. This will be elaborated upon after the statement of Problem II.6.

In this paper we consider the following class of systems:

Definition II.3: An affine control system $\Sigma$ on a full-dimensional polytope $X$ in $\mathbb{R}^{n}$, with $m$ inputs and $p$ outputs is given by

$$
\Sigma:\left\{\begin{array}{l}
\dot{x}(t)=A x(t)+B u(t)+a, \quad x(0)=x_{0} \\
y(t)=C x(t) .
\end{array}\right.
$$

Here $x(t) \in X$ denotes the state, $u(t) \in U$ denotes the input, with $U$ a polytope in $\mathbb{R}^{m}$, called the input polytope, and $y(t) \in$ $Y$ denotes the output, with $Y=\{C x \mid x \in X\}$ a polytope in $\mathbb{R}^{p}$, called the output polytope. In particular, $A \in \mathbb{R}^{n \times n}$, $B \in \mathbb{R}^{n \times m}, a \in \mathbb{R}^{n}$, and $C \in \mathbb{R}^{p \times n}$. Without loss of generality we assume that $C$ is of full row rank; in particular $p \leq n$.

The dynamics described in (1) remain valid as long as the state trajectory $x\left(t, x_{0}\right)$ remains in the state-polytope $X$. Let 
$\mathcal{E} \subset \mathcal{F}_{n-1}(X)$ denote a set of admissible exit facets. In this paper, we want to solve the problem of steering every state trajectory to an admissible exit facet, using static output feedback. In a hybrid systems context this corresponds to the enabling of favorable and the disabling of unfavorable discrete transitions at a given discrete location.

For an exact description of this control problem, we first have to specify which class of output feedback laws we consider.

Definition II.4: Let $\Sigma$ be an affine system on a polytope, with input polytope $U$ and output polytope $Y$. A static output feedback is a Lipschitz-continuous function $g: Y \rightarrow U$ giving input

$$
u(t)=g(y(t))
$$

A static output feedback $g$ is called affine if there exist $H \in$ $\mathbb{R}^{m \times p}$ and $h \in \mathbb{R}^{m}$ such that $g(y)=H y+h$ for all $y \in Y$.

A static output feedback $g$ is called piecewise-affine if there exists a polyhedral subdivision $\left\{Y_{1}, \ldots, Y_{L}\right\}$ of $Y$, matrices $H_{i} \in \mathbb{R}^{m \times p},(i=1, \ldots, L)$ and vectors $h_{i} \in \mathbb{R}^{m},(i=$ $1, \ldots, L)$ such that

$$
\forall i \in\{1, \ldots, L\} \forall y \in Y_{i}: g(y)=H_{i} y+h_{i}
$$

The exact definition of a polyhedral subdivision is provided in Definition III.1 of Section III.

A function $g: Y \rightarrow \mathbb{R}^{m}$ is called admissible as a static output feedback if $g(Y) \subset U$. The closed-loop system of the affine system $\Sigma$ and the static output feedback $g$ is given by

$$
\dot{x}(t)=A x(t)+B g(C x(t))+a, \quad x(0)=x_{0} .
$$

If $g$ is affine, then the closed-loop system (4) will be an autonomous affine system; if $g$ is piecewise-affine, then (4) is an autonomous piecewise-affine system. Note that for every initial state $x_{0} \in X$, (4) will have a unique solution, because $g$ is Lipschitz-continuous.

In this paper, we focus on the following two control problems:

1) Problem II.5 (Blocking Facets): Let $\mathcal{B} \subset \mathcal{F}_{n-1}(X)$ be a subset of the set of facets of state polytope $X$. Find a piecewiseaffine output feedback $g: Y \longrightarrow U$ such that for the closedloop system (4) all facets $F \in \mathcal{B}$ are blocked.

If in Problem II.5, the set $\mathcal{B}$ is equal to $\mathcal{F}_{n-1}(X)$, all facets should be blocked. If output feedback $g$ is a solution to this problem, then all trajectories of the closed-loop system remain in the polytope $X$ forever. In a hybrid systems context this reflects a deadlock in the discrete dynamics: the corresponding discrete location cannot be left, and as soon as the discrete automaton visits this location, no further discrete transitions can occur. Often, the opposite property of guaranteeing liveness of the discrete dynamics is more important for a hybrid system. This means that in every discrete location it is guaranteed that a discrete transition will occur in finite time. For this property one has to verify that all state trajectories leave the state polytope in finite time by crossing an admissible exit facet.

2) Problem II.6 (Control-to-Facets): Let $\mathcal{E} \subset \mathcal{F}_{n-1}(X)$ denote the set of all admissible exit facets. Find a piecewise-affine output feedback $g: Y \longrightarrow U$ such that all state trajectories of the closed-loop system (4) with arbitrary initial state $x(0)=x_{0} \in X$ leave the polytope $X$ in finite time by crossing an admissible exit facet $F \in \mathcal{E}$.

In the control of piecewise-affine hybrid systems on polytopes, a usual control objective is to prevent undesirable switching between certain discrete states. For this one has to guarantee that the continuous state does not leave the polytope through a nonadmissible facet. However, it is possible that the exit point belongs to several facets. In the approach to the control of hybrid systems described in [11], it is assumed that in that case only those events can occur, that correspond to facets that are crossed. Since, by definition, blocked facets cannot be crossed, the control objective can be restated as to block all nonadmissible facets (Problem II.5, with $\mathcal{B}=\mathcal{F}_{n-1}(X) \backslash \mathcal{E}$ ). In this situation, it is still possible that an exit point belongs to the intersection of a blocked and a nonblocked facet, but no trajectory will cut the blocked facet transversely, and the event corresponding to the blocked facet will not occur.

Note that blocking all nonadmissible exit facets is not sufficient for solving Problem II.6. If a closed-loop system admits both trajectories that leave $X$ in finite time and trajectories that remain in $X$ forever, then the corresponding output feedback law $g$ does not solve Problem II.6, because it does not guarantee departure from polytope $X$ in finite time.

Proposition II.7: A piecewise-affine static output feedback $g: Y \longrightarrow U$ solves Control Problem II.6 for the nonempty set $\mathcal{E}$ of admissible exit facets if and only if the following two conditions are satisfied:

(i) the output feedback $g$ solves Problem II.5 for the set $\mathcal{B}=$ $\mathcal{F}_{n-1}(X) \backslash \mathcal{E}$

(ii) all state trajectories of closed-loop system (4) leave the polytope $X$ in finite time.

Proof: Assume that $g: Y \longrightarrow U$ is an output feedback that solves Problem II.6. Then condition (ii) is satisfied, so it suffices to prove (i). Let $F \in \mathcal{F}_{n-1}(X) \backslash \mathcal{E}$, and let $\hat{x} \in F$. Then $\hat{x} \notin \operatorname{Ex}(F)$, because the trajectory $x(t, \hat{x})$ does not cross $F$. Hence, $\operatorname{Ex}(F)=\varnothing$, and indeed facet $F$ is blocked with respect to closed-loop system (4).

Assume that $g: Y \longrightarrow U$ is an output feedback that satisfies conditions (i) and (ii). According to (i), all facets $F \in$ $\mathcal{F}_{n-1}(X) \backslash \mathcal{E}$ are blocked with respect to the closed-loop dynamics, and blocked facets cannot be crossed. At the same time, (ii) states that all trajectories of closed-loop system (4) leave polytope $X$ in finite time. Furthermore, [11, Lemma 4.7] implies that a trajectory that leaves $X$ in finite time, crosses at least one facet. Hence, it must cross a facet $F \in \mathcal{E}$.

In [9] and [11], control-to-facet problems similar to Problems II.5 and II.6 have been solved using state- instead of output feedback. First a necessary condition and a sufficient condition for solvability are obtained, formulated as sets of linear inequalities on the inputs at the vertices of the state polytope $X$. The second step is to realize these inputs at the vertices by an admissible piecewise-affine state feedback law. Since the state polytope is convex, and the closed-loop system piecewise-affine, this approach guarantees that on all nonadmissible exit-facets the vector field of the closed-loop system is pointing into the state polytope. Additionally one can check that the state polytope is left in finite time. For the construction of the state feedback law, a triangulation of the state polytope is used. There 
are no special requirements on this triangulation, because the solvability conditions are completely independent of the chosen triangulation.

For our solution of the output feedback Problems II.5 and II.6, we use a similar approach. This problem with partial observations is more difficult, because one observed output $y \in Y$ corresponds to a set of states $X_{y}=\{x \in X \mid C x=y\}$. The set $X_{y}$ may intersect several facets of $X$, so the corresponding input $u=g(y)$ has to satisfy constraints with respect to all these facets at the same time. For this purpose we first construct a special polyhedral subdivision of the output polytope, that meets certain compatibility conditions with the state polytope and the output map. Section III is devoted to this subject. Next, we set up a system of linear inequalities for the inputs at each vertex of this polyhedral subdivision. If these inequalities have a solution, a piecewise-affine output feedback is obtained by refining the subdivision to a triangulation, and interpolating the inputs at the vertices of the triangulation. This part of the construction will be elaborated in Section IV.

\section{Subdivision of the Output Polytope}

\section{A. Polyhedral Complexes}

In order to handle the different constraints on the inputs, and to construct a piecewise-affine output feedback, we need a subdivision of the output polytope with special properties. For this we introduce in this section the so-called chamber complex (see, e.g., [16] and [17]). Using this subdivision, one may obtain a necessary and sufficient condition for the solvability of Problem II.5 and a sufficient condition for the solvability of Problem II.6. The construction of the control law will be based on a triangulation that refines the chamber complex.

Definition III.1: A polyhedral complex $\mathcal{P}$ is a finite collection of polytopes that satisfies the following two conditions:

1) if $P \in \mathcal{P}$, then $F \in \mathcal{P}$ for all faces $F$ of $P$,

2) if $P, Q \in \mathcal{P}$, then either $P \cap Q$ is a face of both $P$ and $Q$, or $P \cap Q=\varnothing$.

The underlying space of $\mathcal{P}$ is $|\mathcal{P}|:=\bigcup_{P \in \mathcal{P}} P$. A polyhedral complex $\mathcal{P}$ is a called a polyhedral subdivision of a polytope $X$ if $X=|\mathcal{P}|$. A polyhedral complex $\mathcal{Q}$ is called a refinement of polyhedral complex $\mathcal{P}$ if

$$
\forall P \in \mathcal{P}: P=\bigcup\{Q \in \mathcal{Q} \mid Q \subset P\} .
$$

A polytope of dimension $k$ with $k+1$ vertices is called a $k$-simplex. A polyhedral subdivision $\mathcal{T}$ of polytope $X$ is called a triangulation of $X$ if every $S \in \mathcal{T}$ is a simplex. The vertex set of a polyhedral complex $\mathcal{P}$ is the union of all polytopes $P \in \mathcal{P}$ that consist of one point, and is denoted $\mathcal{V}(\mathcal{P})$.

In the sequel we will need a subdivision of the output polytope $Y=h(X)$, such that it satisfies a compatibility condition with the state polytope $X$ and the output map $h$ :

Definition III.2: Let $X$ be a full-dimensional polytope in $\mathbb{R}^{n}$, and let $Y=h(X)$, with $h: \mathbb{R}^{n} \rightarrow \mathbb{R}^{p}$ a surjective linear map, i.e., $h(x)=C x$, where $C$ is a $p \times n$ matrix of rank $p$. A polyhedral subdivision $\mathcal{P}$ of polytope $Y$ is called $(X, h)$-compatible if

$$
\forall P \in \mathcal{P}, \forall x \in \mathcal{V}\left(h^{-1}(P) \cap X\right): h(x) \in \mathcal{V}(P)
$$

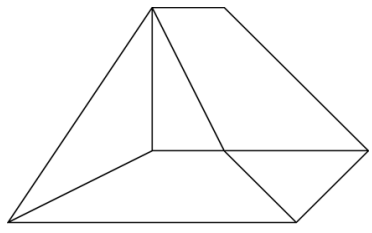

(a)

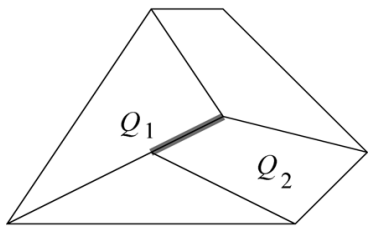

(b)

Fig. 1. (a) Polyhedral subdivision of a polytope in $\mathbb{R}^{2}$ into three triangles and two quadrangles; (b) subdivision of the same polytope into polyhedra which do not form a polyhedral complex. The intersection of $Q_{1}$ and $Q_{2}$ is not a face of $Q_{1}$.

where $h^{-1}(W):=\left\{x \in \mathbb{R}^{n} \mid h(x) \in W\right\}$ denotes the inverse image of a set $W \subset \mathbb{R}^{p}$ under $h$.

In words: a polyhedral complex $\mathcal{P}$ of $Y$ is $(X, h)$-compatible, if for every polytope $P \in \mathcal{P}$, the vertices of the polytope $h^{-1}(P) \cap X$ are mapped to vertices of $P$ by the mapping $h$.

Note that the compatibility notion for subdivisions introduced in $[15, \mathrm{Ch} .9]$ is different from the one described in Definition III.2. Therefore, the results from [15, Ch. 9] are not directly applicable in the context of this paper.

The compatibility condition (5) can be stated in several equivalent ways.

Lemma III.3: Let $X$ be a full-dimensional polytope in $\mathbb{R}^{n}$, and $h: \mathbb{R}^{n} \rightarrow \mathbb{R}^{p}$ be a surjective linear map. A polyhedral subdivision $\mathcal{P}$ of polytope $Y=h(X)$ is $(X, h)$-compatible if and only if one of the following equivalent conditions holds:

(i) $\forall P \in \mathcal{P}: h^{-1}(P) \cap X=\operatorname{conv}\left(h^{-1}(\mathcal{V}(P)) \cap X\right)$;

(ii) $\forall P \in \mathcal{P}: h\left(\mathcal{V}\left(h^{-1}(P) \cap X\right)\right)=\mathcal{V}(P)$,

where $\operatorname{conv}(S)$ denotes the convex hull of $S$.

Proof:

Equation (5) $\Longrightarrow($ i): Assume that (5) holds, and let $P \in \mathcal{P}$. Then $\mathcal{V}\left(h^{-1}(P) \cap X\right) \subset h^{-1}(\mathcal{V}(P)) \cap X$, and by taking convex hulls we obtain $h^{-1}(P) \cap X \subset$ $\operatorname{conv}\left(h^{-1}(\mathcal{V}(P)) \cap X\right)$. To prove the opposite inclusion, let $x \in \operatorname{conv}\left(h^{-1}(\mathcal{V}(P)) \cap X\right)$. Then there exist $k \in \mathbb{N}$, $v_{1}, \ldots, v_{k} \in h^{-1}(\mathcal{V}(P)) \cap X$, and $\lambda_{1}, \ldots, \lambda_{k} \in[0,1]$ such that $\sum_{i=1}^{k} \lambda_{i} v_{i}=x$ and $\sum_{i=1}^{k} \lambda_{i}=1$. In particular, $v_{1}, \ldots, v_{k} \in X$ and $h\left(v_{1}\right), \ldots, h\left(v_{k}\right) \in \mathcal{V}(P)$. Since $X$ and $P$ are polytopes, and $h$ is a linear map, it follows that $x=\sum_{i=1}^{k} \lambda_{i} v_{i} \in X$, and $h(x)=\sum_{i=1}^{k} \lambda_{i} h\left(v_{i}\right) \in P$, so $x \in h^{-1}(P) \cap X$.

(i) $\Longrightarrow$ (5): Assume that (i) holds, and let $P \in \mathcal{P}$. Then (i) implies that

$$
\begin{aligned}
\mathcal{V}\left(h^{-1}(P)\right. & \cap X) \\
& \left.=\mathcal{V}\left(\operatorname{conv}\left(h^{-1}(\mathcal{V}(P)) \cap X\right)\right)\right) \subset h^{-1}(\mathcal{V}(P)) \cap X .
\end{aligned}
$$

Hence, if $x \in \mathcal{V}\left(h^{-1}(P) \cap X\right)$, then $x \in h^{-1}(\mathcal{V}(P)) \cap X$, i.e., $x \in X$, and $h(x) \in \mathcal{V}(P)$.

Equation (5) $\Longrightarrow$ (ii): Assume that (5) holds, and let $P \in \mathcal{P}$. From (5) the inclusion $h\left(\mathcal{V}\left(h^{-1}(P) \cap X\right)\right) \subset \mathcal{V}(P)$ is trivial, so it suffices to prove the inclusion in the opposite 
direction. Since $P \subset h(X)$, we have $P=h\left(h^{-1}(P) \cap X\right)$, and by only considering the vertices of $P$ we obtain

$$
\mathcal{V}(P)=\mathcal{V}\left(h\left(h^{-1}(P) \cap X\right)\right) \subset h\left(\mathcal{V}\left(h^{-1}(P) \cap X\right)\right) .
$$

(ii) $\Longrightarrow$ (5): Assume that (ii) holds. Let $P \in \mathcal{P}$, and $x \in$ $\mathcal{V}\left(h^{-1}(P) \cap X\right)$. According to (ii) we have $h(x) \in \mathcal{V}(P)$.

The next lemma states that in a compatible subdivision $\mathcal{P}$, the vertices of the inverse image of a polytope $P \in \mathcal{P}$ coincide with the vertices of the inverse images of the single vertices of $P$.

Lemma III.4: Let $X$ be a full-dimensional polytope in $\mathbb{R}^{n}$, and $h: \mathbb{R}^{n} \rightarrow \mathbb{R}^{p}$ be a surjective linear map. Let $\mathcal{P}$ be an $(X, h)$-compatible polyhedral subdivision of polytope $Y=h(X)$. Then for all $P \in \mathcal{P}$ we have

$$
\mathcal{V}\left(h^{-1}(P) \cap X\right)=\bigcup_{w \in \mathcal{V}(P)} \mathcal{V}\left(h^{-1}(w) \cap X\right) .
$$

Proof:

" $\subset$ ": Let $x \in \mathcal{V}\left(h^{-1}(P) \cap X\right)$. Since subdivision $\mathcal{P}$ is $(X, h)$-compatible, we have $w:=h(x) \in \mathcal{V}(P)$. So, obviously $x \in h^{-1}(w) \cap X$, and there exist $k \in \mathbb{N}$, $v_{1}, \ldots, v_{k} \in \mathcal{V}\left(h^{-1}(w) \cap X\right)$ and $\lambda_{1}, \ldots, \lambda_{k} \in(0,1]$ such that $x=\sum_{i=1}^{k} \lambda_{i} v_{i}$ and $\sum_{i=1}^{k} \lambda_{i}=1$. For $i=1, \ldots, k$ we have $v_{i} \in h^{-1}(w) \cap X$; hence, $h\left(v_{i}\right)=w \in \mathcal{V}(P) \subset$ $P$. So, in particular $v_{i} \in h^{-1}(P) \cap X$ for $i=1, \ldots, k$. Since $x$ is a vertex of $h^{-1}(P) \cap X$, and $x=\sum_{i=1}^{k} \lambda_{i} v_{i}$, it follows that $v_{i}=x$ for all $i=1, \ldots, k$. Hence, $x=v_{i} \in$ $\mathcal{V}\left(h^{-1}(w) \cap X\right)$.

' $\supset$ ': Let $x \in \mathcal{V}\left(h^{-1}(w) \cap X\right)$ for some $w \in \mathcal{V}(P)$. Then in particular $x \in X$ and $h(x)=w \in \mathcal{V}(P) \subset P$, so $x \in h^{-1}(P) \cap X$. There exist $k \in \mathbb{N}, v_{1}, \ldots, v_{k} \in$ $\mathcal{V}\left(h^{-1}(P) \cap X\right)$ and $\lambda_{1}, \ldots, \lambda_{k} \in(0,1]$ such that $x=$ $\sum_{i=1}^{k} \lambda_{i} v_{i}$ and $\sum_{i=1}^{k} \lambda_{i}=1$. Since polyhedral subdivision $\mathcal{P}$ is $(X, h)$-compatible, and for all $i=1, \ldots, k$ we have $v_{i} \in \mathcal{V}\left(h^{-1}(P) \cap X\right)$, it follows from Lemma III.3 (ii) that $h\left(v_{i}\right) \in \mathcal{V}(P)$ for $i=1, \ldots, k$. Furthermore, $h$ is linear, so

$$
w=h(x)=h\left(\sum_{i=1}^{k} \lambda_{i} v_{i}\right)=\sum_{i=1}^{k} \lambda_{i} h\left(v_{i}\right)
$$

i.e., $w \in \mathcal{V}(P)$ is a convex combination of $h\left(v_{i}\right),(i=$ $1, \ldots, k)$. Since $w$ is a vertex of $P$ and also $h\left(v_{i}\right) \in \mathcal{V}(P)$, $(i=1, \ldots, k)$ we must have that $h\left(v_{i}\right)=w$ for all $i=$ $1, \ldots, k$. In particular, $v_{1}, \ldots, v_{k} \in h^{-1}(w) \cap X$, and $x$ is both a vertex of $h^{-1}(w) \cap X$ and a convex combination of $v_{1}, \ldots, v_{k}$. We conclude that $v_{i}=x,(i=1, \ldots, k)$; hence, $x \in \mathcal{V}\left(h^{-1}(P) \cap X\right)$.

Property (6) is of particular interest for polytopes $P$ that are simplices. Any point $y$ of a simplex $S$ can be uniquely represented as a convex combination of the vertices of $S$. If (6) is satisfied for simplex $S$, then the next lemma states that every state $x \in h^{-1}(y) \cap X$ can be written as exactly the same convex combination of certain states $z \in X$, whose corresponding outputs $h(z)$ are vertices of $S$. The exact statement of the result is as follows:
Lemma III.5: Suppose that $X$ is a full-dimensional polytope in $\mathbb{R}^{n}$, and $h: \mathbb{R}^{n} \rightarrow \mathbb{R}^{p}$ is a surjective linear map. Let $S$ be a $k$-dimensional simplex $(k \leq p)$ contained in $Y=h(X)$, with vertex set $\mathcal{V}(S)=\left\{w_{1}, \ldots, w_{k+1}\right\}$. Assume that

$$
\mathcal{V}\left(h^{-1}(S) \cap X\right)=\bigcup_{i=1}^{k+1} \mathcal{V}\left(h^{-1}\left(w_{i}\right) \cap X\right) .
$$

Let $\lambda_{1}, \ldots, \lambda_{k+1} \in(0,1]$ be such that $\sum_{i=1}^{k+1} \lambda_{i}=1$, and define $\hat{y} \in S$ by $\hat{y}=\sum_{i=1}^{k+1} \lambda_{i} w_{i}$. Then

(i) For all $\hat{x} \in h^{-1}(\hat{y}) \cap X$, there exist $z_{i} \in h^{-1}\left(w_{i}\right) \cap X$, $(i=1, \ldots, k+1)$ such that $\hat{x}=\sum_{i=1}^{k+1} \lambda_{i} z_{i}$.

(ii) If $\hat{x} \in h^{-1}(\hat{y}) \cap X$ belongs to a particular facet $F$ of $X$, then for all $i=1, \ldots, k+1$, the point $z_{i}$ in (i) belongs to $h^{-1}\left(w_{i}\right) \cap F$.

Proof:

(i) Let $\hat{x} \in h^{-1}(\hat{y}) \cap X$. Since $h(\hat{x})=\hat{y} \in S$, it is obvious that $\hat{x} \in h^{-1}(S) \cap X$, so $\hat{x}$ is a convex combination of the vertices of $h^{-1}(S) \cap X$. According to (7), $\hat{x}$ is a convex combination of points in $\bigcup_{i=1}^{k+1} \mathcal{V}\left(h^{-1}\left(w_{i}\right) \cap X\right)$. For $i=1, \ldots, k+1$ we denote $\mathcal{V}\left(h^{-1}\left(w_{i}\right) \cap X\right)=$ $\left\{v_{i, 1}, \ldots, v_{i, \ell_{i}}\right\}$. Then there exist scalars $\rho_{1,1}, \ldots, \rho_{1, \ell_{1}}$, $\rho_{2,1}, \ldots, \rho_{2, \ell_{2}}, \ldots, \rho_{k+1,1}, \ldots, \rho_{k+1, \ell_{k+1}} \in[0,1]$ such that:
1) $\sum_{i=1}^{k+1} \sum_{j=1}^{\ell_{i}} \rho_{i, j} v_{i, j}=\hat{x}$;
2) $\sum_{i=1}^{k+1} \sum_{j=1}^{\ell_{i}} \rho_{i, j}=1$

For $i=1, \ldots, k+1$ we define $\mu_{i}:=\sum_{j=1}^{\ell_{i}} \rho_{i, j}$. Then $\mu_{i} \in[0,1]$ and $\sum_{i=1}^{k+1} \mu_{i}=1$. Subsequently we define for $i=1, \ldots, k+1$ and $j=1, \ldots, \ell_{i}$

$$
\alpha_{i, j}= \begin{cases}0, & \text { if } \mu_{i}=0 \\ \frac{\rho_{i, j}}{\mu_{i}}, & \text { if } \mu_{i} \neq 0 .\end{cases}
$$

Then $\alpha_{i, j} \in[0,1]$, and, if $\mu_{i} \neq 0$, then $\sum_{j=1}^{\ell_{i}} \alpha_{i, j}=1$. Furthermore

$$
\begin{aligned}
\hat{x} & =\sum_{i=1}^{k+1} \sum_{j=1}^{\ell_{i}} \rho_{i, j} v_{i, j}=\sum_{i=1, \mu_{i} \neq 0}^{k+1} \mu_{i} \sum_{j=1}^{\ell_{i}} \frac{\rho_{i, j}}{\mu_{i}} v_{i, j} \\
& =\sum_{i=1}^{k+1} \mu_{i} \sum_{j=1}^{\ell_{i}} \alpha_{i, j} v_{i, j} .
\end{aligned}
$$

For $i=1, \ldots, k+1$, we define $z_{i}=\sum_{j=1}^{\ell_{i}} \alpha_{i, j} v_{i, j}$. Then $\mu_{i}=0$ implies $z_{i}=0$, and $\mu_{i} \neq 0$ implies that $z_{i} \in \operatorname{conv}\left(\mathcal{V}\left(h^{-1}\left(w_{i}\right) \cap X\right)\right)=h^{-1}\left(w_{i}\right) \cap X$, so in particular $h\left(z_{i}\right)=w_{i}$. Since the output map $h$ is linear, it follows that

$$
\hat{y}=h(\hat{x})=h\left(\sum_{i=1}^{k+1} \mu_{i} z_{i}\right)=\sum_{i=1, \mu_{i} \neq 0}^{k+1} \mu_{i} h\left(z_{i}\right)=\sum_{i=1}^{k+1} \mu_{i} w_{i} .
$$

On the other hand, since $S$ is a $k$-dimensional simplex, $\hat{y}=\sum_{i=1}^{k+1} \lambda_{i} w_{i}$ is the unique representation of $\hat{y}$ as convex combination of $w_{1}, \ldots, w_{k+1}$. It follows that $\mu_{i}=\lambda_{i} \neq 0$ for $i=1, \ldots, k+1$. We conclude that

$$
\hat{x}=\sum_{i=1}^{k+1} \lambda_{i} z_{i}
$$

with $z_{i} \in h^{-1}\left(w_{i}\right) \cap X,(i=1, \ldots, k+1)$. 
(ii) Suppose that $\hat{x} \in F$, for some facet $F$ of $X$. Let $n_{F}$ denote a normal vector of $F$ pointing out of $X$. Then there exists $\alpha_{F} \in \mathbb{R}$ such that

$$
\begin{array}{ll}
\forall x \in X: & n_{F}^{T} x \leq \alpha_{F} \\
\forall x \in F: & n_{F}^{T} x=\alpha_{F} .
\end{array}
$$

Clearly $n_{F}^{T} \hat{x}=\alpha_{F}$ because $\hat{x} \in F$. Suppose that there exists an $i \in\{1, \ldots, k+1\}$ such that $z_{i} \notin F$. Then $n_{F}^{T} z_{i}<\alpha_{F}$, and we obtain

$$
n_{F}^{T} \hat{x}=\sum_{i=1}^{k+1} \lambda_{i} n_{F}^{T} z_{i}<\sum_{i=1}^{k+1} \lambda_{i} \alpha_{F}=\alpha_{F}
$$

which contradicts the fact that $n_{F}^{T} \hat{x}=\alpha_{F}$.

If $\mathcal{T}$ is a so-called compatible triangulation of the output polytope, i.e., $\mathcal{T}$ is a compatible polyhedral subdivision and a triangulation at the same time, then Lemma III.4 and Lemma III.5 imply that the properties described in Lemma III.5 hold in every simplex of triangulation $\mathcal{T}$. In Section IV, this observation will play a prominent role in the proof of our solution to the facet-blocking problem using piecewise-affine output feedback. However, before turning to this main result, we have to investigate how compatible triangulations can be obtained.

\section{B. Construction of a Compatible Triangulation}

In order to generate a compatible subdivision, we start with the so-called chamber complex of $(X, h)$ as defined in, e.g., [17].

Definition III.6: Let $X$ be an polyhedron in $\mathbb{R}^{n}, h: \mathbb{R}^{n} \rightarrow \mathbb{R}^{p}$ a surjective linear map, and $Y=h(X)$. The chamber complex $\mathcal{C}$ of $(X, h)$ is defined as the collection of polytopes $\{\sigma(y) \mid y \in$ $Y$ \}, where

$$
\sigma(y)=\bigcap\{h(F) \mid F \text { is a face of } X \text { and } y \in h(F)\}
$$

is the chamber of $y \in Y$.

Note that the chamber complex is a polyhedral subdivision of the output polytope $Y=h(X)$.

The next result states that the chamber complex is indeed a polyhedral complex that combines the properties we are interested in.

Theorem III.7: The chamber complex $\mathcal{C}$ of $(X, h)$ is an $(X, h)$-compatible polyhedral subdivision of the polytope $Y=h(X)$.

The fact that the chamber complex is a polyhedral subdivision of $Y$ is stated, but not proved, in [17]. Using [17, Prop. 2.4] one may also show that this polyhedral subdivision is $(X, h)$-compatible, but again, no proof is given. Because of the fundamental importance of Theorem III.7, we provide a complete proof in the Appendix. Theorem A.11 states that the chamber complex is a polyhedral subdivision, and compatibility is an immediate consequence of Theorem A.12.

The construction of the chamber complex of $Y=h(X)$ is relatively straightforward. Let $\mathcal{H}_{p}$ denote the collection of faces $F$ of $X$ such that $\operatorname{dim} F=\operatorname{dim} h(F)=p$, where $p=\operatorname{dim} Y$. For every subset $\mathcal{G} \subset \mathcal{H}_{p}$, we define $C_{\mathcal{G}}:=\bigcap_{F \in \mathcal{G}} h(F)$. The set of all such faces $C_{\mathcal{G}}$, with $\mathcal{G}$ any subset of $\mathcal{H}_{p}$ such that $C_{\mathcal{G}}$ is nonempty, forms the chamber complex of $Y$. Indeed, if $\operatorname{dim} h(F)<\operatorname{dim} F$, then $h(F)=h(\partial F)$, where $\partial F$ denotes the boundary of $F$ [see Lemma A.4 (2)]; hence, $h(F)=\cup\{h(G) \mid G$ is a facet of $F\}$. So, the full-dimensional chambers must be intersections of faces $F$ of $X$ such that $\operatorname{dim} F=\operatorname{dim} h(F)=\operatorname{dim} Y$. In practice, it often suffices to compute all full-dimensional chambers, which are given by chambers $C_{\mathcal{G}}$ for which $\bigcap_{F \in \mathcal{G}} h(\operatorname{relint}(F))$ is nonempty. An illustrative example of this approach is given in Section V, Example V.2.

The next result states that any refinement of the chamber complex is still an $(X, h)$-compatible subdivision of $Y=h(X)$.

Theorem III.8: Let $\mathcal{P}$ be a polyhedral subdivision of $Y$ which refines the chamber complex $\mathcal{C}$ of $(X, h)$. Then $\mathcal{P}$ is $(X, h)$ compatible.

For the purpose of constructing a static output feedback law, we are particularly interested in polyhedral subdivisions of the output polytope that are both triangulations and $(X, h)$-compatible. The existence of a triangulation of a given polyhedral subdivision is a well-known result in the literature (see [15] for a recent monograph on triangulations). A simple construction is the lexicographic triangulation (see, e.g., [12, Section 2] and references therein):

Theorem III.9: Let $\mathcal{P}$ be a polyhedral subdivision of the polytope $P$. Then there exists a triangulation $\mathcal{T}$ that refines $\mathcal{P}$. Moreover, $\mathcal{T}$ can be chosen so that $\mathcal{V}(\mathcal{T})=\mathcal{V}(\mathcal{P})$.

Finally, triangulations of the chamber complex turn out to be the only $(X, h)$-compatible triangulations of the output polytope $Y=h(X)$.

Theorem III.10: Let $\mathcal{T}$ be a triangulation of the polytope $Y=$ $h(X)$. Then $\mathcal{T}$ is an $(X, h)$-compatible triangulation of $Y$ if and only if $\mathcal{T}$ is a refinement of the chamber complex $\mathcal{C}$ of $(X, h)$.

Theorem III.8 and Theorem III.10 are combined in Theorem A.12, a complete proof of which is given in the Appendix.

At this point, it is straightforward how an $(X, h)$-compatible triangulation of the output polytope $Y=h(X)$ may be constructed. The first step is to construct the chamber complex $\mathcal{C}$ of $(X, h)$. This can be done by computing intersections of $h(F)$ for all faces $F \in \mathcal{F}_{p}(X)$, with $p=\operatorname{dim} Y$. In the second step this chamber complex is triangulated in such a way that $\mathcal{V}(\mathcal{T})=\mathcal{V}(\mathcal{C})$. According to Theorem III.8 or Theorem III.10, this triangulation will be $(X, h)$-compatible.

\section{SOlVABILITy CONDITIONS AND CONSTRUCTION OF A PIECEWISE-AfFine OUTPUT FEEDBACK}

If $\mathcal{T}$ is an $(X, h)$-compatible triangulation of the output polytope $Y$, and if for every vertex $w \in \mathcal{V}(\mathcal{T})$ a corresponding input $u_{w} \in U$ is fixed, then an admissible piecewise-affine output feedback is easily constructed. For every $y \in Y$, let $S_{y} \in \mathcal{T}$ denote the simplex in $\mathcal{T}$ of smallest dimension, that contains the point $y$. Then $y$ can be written in a unique way as a convex combination of the vertices of $S_{y}$

$$
y=\sum_{w \in \mathcal{V}\left(S_{y}\right)} \lambda_{y, w} w
$$

with $\lambda_{y, w} \in(0,1]$ for all $w \in \mathcal{V}\left(S_{y}\right)$, and $\sum_{w \in \mathcal{V}\left(S_{y}\right)} \lambda_{y, w}=1$. Next we define the output feedback $g: Y \longrightarrow U$ by

$$
g(y)=\sum_{w \in \mathcal{V}\left(S_{y}\right)} \lambda_{y, w} u_{w} .
$$


Then $g$ is a continuous function, and affine on every simplex of $\mathcal{T}$. Furthermore, $g(w)=u_{w}$ for all $w \in \mathcal{V}(\mathcal{T})$.

The key idea behind our approach to solve Problems II.5 and II.6 is to choose the inputs $u_{w},(w \in \mathcal{V}(\mathcal{T}))$ in such a way that in all states $x \in X$ corresponding to output $w$, the vector $A x+B u_{w}+a$ is pointing in a direction, that locally fulfills the control objective. Since the dynamics of the system is convex, an inequality on the inner product of a fixed vector $n$ and the vector field $A x+B g(C x)+a$ of the closed-loop system that is valid in all vertices of a facet of $X$, extends to all points of the facet. Furthermore, since the triangulation $\mathcal{T}$ of $Y$ is $(X, h)$-compatible, it is a refinement of the chamber complex $\mathcal{C}$ of $(X, h)$, and every vertex $v$ of $X$ corresponds to a vertex $w=h(v)$ of $\mathcal{C}$. Combining these observations, it is possible to formulate a necessary and sufficient condition for solvability of Problem II.5 and a sufficient condition for solvability of Problem II.6 in terms of a set of linear inequalities on the inputs corresponding to the vertices of the chamber complex $\mathcal{C}$ of $(X, h)$. The exact statement is given in the following theorem:

Theorem IV.1: Consider an affine system (1) on a full-dimensional polytope $X$ in $\mathbb{R}^{n}$. Let $\mathcal{E} \subset \mathcal{F}_{n-1}(X)$ be a set of admissible exit facets, and let $h: \mathbb{R}^{n} \rightarrow \mathbb{R}^{p}$ denote the linear output map $h(x)=C x$, with $\operatorname{rank}(C)=p$. Let $\mathcal{C}$ be the chamber complex of $(X, h)$. For all $w \in \mathcal{V}(\mathcal{C})$ we define the set of inputs that satisfy all relevant linear inequalities at output $w$ by

$$
\begin{array}{r}
Q_{w}:=\left\{u \in U \mid \forall v \in \mathcal{V}\left(h^{-1}(w) \cap X\right), \forall F \in \mathcal{F}_{n-1}(v, X) \backslash \mathcal{E}:\right. \\
\left.n_{F}^{T}(A v+B u+a) \leq 0\right\} \quad(10)
\end{array}
$$

where $n_{F}$ denotes the unit normal vector of facet $F$, pointing out of polytope $X$. Then

(i) Problem II.5 is solvable if and only if $Q_{w} \neq \varnothing$ for all $w \in \mathcal{V}(\mathcal{C})$

(ii) Problem II.6 is solvable if there exists a direction $n_{D} \in$ $\mathbb{R}^{n}$ such that

$$
\begin{gathered}
\forall w \in \mathcal{V}(\mathcal{C}), \exists u_{w} \in \mathcal{V}\left(Q_{w}\right), \quad \forall v \in \mathcal{V}\left(h^{-1}(w) \cap X\right): \\
n_{D}{ }^{T}\left(A v+B u_{w}+a\right)>0 .
\end{gathered}
$$

Proof:

(i) Let $g: Y \longrightarrow U$ be a piecewise-affine output feedback, solving Problem II.5. We first show that $Q_{w} \neq \varnothing$ for all $w \in \mathcal{V}(\mathcal{C})$, i.e., the necessity of condition (i).

Let $w \in \mathcal{V}(\mathcal{C})$, and define $u_{w}:=g(w)$. We will show that $u_{w} \in Q_{w}$, i.e.,

$$
\begin{aligned}
\forall v \in \mathcal{V}\left(h^{-1}(w) \cap X\right), \quad \forall F \in \mathcal{F}_{n-1}(v, X) \backslash \mathcal{E}: \\
n_{F}^{T}\left(A v+B u_{w}+a\right) \leq 0 .
\end{aligned}
$$

Let $v \in \mathcal{V}\left(h^{-1}(w) \cap X\right)$. Then $v \in X$ and $h(v)=$ $w$, so the output corresponding to state $v$ is $w$. Let $F \in$ $\mathcal{F}_{n-1}(v, X) \backslash \mathcal{E}$. The closed-loop system is described by

$$
\dot{x}(t)=A x(t)+B g(h(x(t)))+a .
$$

Since the output-feedback $g: Y \longrightarrow U$ solves Problem II.5, the direction of the vector field of the closed-loop system in the point $v$ does not have a positive component in the direction of $n_{F}$

$$
\left.n_{F}^{T} \dot{x}\right|_{v}=n_{F}^{T}(A v+B g(h(v))+a) \leq 0 .
$$

Since $h(v)=w$ and $g(w)=u_{w}$, we have

$$
n_{F}^{T}\left(A v+B u_{w}+a\right) \leq 0
$$

and indeed (12) holds.

Next, assume that $Q_{w} \neq \varnothing$ for all $w \in \mathcal{V}(\mathcal{C})$. We will show that Problem II.5 is solvable, i.e., the sufficiency of condition (i).

First we fix for every point $w \in \mathcal{V}(\mathcal{C})$ an input $u_{w} \in Q_{w}$. Let $\mathcal{T}$ be a triangulation of the chamber complex $\mathcal{C}$ of $(X, h)$ with the property that $\mathcal{V}(\mathcal{T})=\mathcal{V}(\mathcal{C})$, cf. Theorem III.9; in particular $\mathcal{T}$ is an $(X, h)$-compatible triangulation of the output polytope $Y=h(X)$. Let $g: Y \longrightarrow U$ be the piecewise-affine output feedback, corresponding to inputs $\left\{u_{w} \in U \mid w \in \mathcal{V}(\mathcal{C})\right\}$ and triangulation $\mathcal{T}$, as defined in (9). We will prove that the feedback law $u=g(y)$ is a solution for Problem II.5, by verifying that

$\forall F \in \mathcal{F}_{n-1}(X) \backslash \mathcal{E}, \forall x \in F: n_{F}^{T}(A x+B g(h(x))+a) \leq 0$.

Let $\hat{F} \in \mathcal{F}_{n-1}(X) \backslash \mathcal{E}$, and let $\hat{x} \in \hat{F}$. Define $\hat{y}:=h(\hat{x})$. Let $\left\{w_{1}, \ldots, w_{k+1}\right\}$ (with $k \leq p$ ) be the set of vertices of simplex $S_{\hat{y}}$, i.e., the simplex in $\mathcal{T}$ of smallest dimension, that contains $\hat{y}$. Since $\hat{y}$ can be written in a unique way as a convex combination of vertices of $S_{\hat{y}}$, there exist unique $\lambda_{1}, \ldots, \lambda_{k+1} \in(0,1]$ such that:

(1) $\sum_{i=1}^{k+1} \lambda_{i} w_{i}=\hat{y}$

(2) $\sum_{i=1}^{k+1} \lambda_{i}=1$.

Output feedback law (9) fixes the input $\hat{u}$ corresponding to output $\hat{y}$ by

$$
\hat{u}=g(\hat{y})=\sum_{i=1}^{k+1} \lambda_{i} u_{w_{i}} .
$$

We will show that this input $\hat{u}$ guarantees that

$$
n_{\hat{F}}^{T}(A \hat{x}+B \hat{u}+a) \leq 0 .
$$

Since $\hat{x} \in X$ and $\hat{y}=h(\hat{x}) \in S_{\hat{y}}$, it is clear that $\hat{x} \in h^{-1}(\hat{y}) \cap X$. Furthermore, since triangulation $\mathcal{T}$ is $(X, h)$-compatible, Lemma III.4 implies that $\mathcal{V}\left(h^{-1}\left(S_{\hat{y}}\right) \cap X\right)=\cup_{i=1}^{k+1} \mathcal{V}\left(h^{-1}\left(w_{i}\right) \cap X\right)$. So, according to Lemma III.5 (ii), and using the fact that $\hat{x} \in \hat{F}$, there exist $z_{i} \in h^{-1}\left(w_{i}\right) \cap \hat{F},(i=1, \ldots, k+1)$, such that $\hat{x}=\sum_{i=1}^{k+1} \lambda_{i} z_{i}$.

Recall that for all $i=1, \ldots, k+1: u_{w_{i}} \in Q_{w_{i}}$, and that $\hat{F}$ is a facet of $X$, not belonging to $\mathcal{E}$. So, according to (10), we have for each $i \in\{1, \ldots, k+1\}$

$$
\forall v \in \mathcal{V}\left(h^{-1}\left(w_{i}\right) \cap X\right) \cap \hat{F}: n_{\hat{F}}^{T}\left(A v+B u_{w_{i}}+a\right) \leq 0 \text {. }
$$

Since $z_{i} \in h^{-1}\left(w_{i}\right) \cap X$ and $z_{i} \in \hat{F}, z_{i}$ can be written as a convex combination of vectors $v \in \mathcal{V}\left(h^{-1}\left(w_{i}\right) \cap X\right) \cap \hat{F}$. In combination with (13) this implies that

$\forall i \in\{1, \ldots, k+1\}: n_{\hat{F}}^{T}\left(A z_{i}+B u_{w_{i}}+a\right) \leq 0$. 
We conclude that in state $\hat{x} \in \hat{F}$ the vector field of the closed-loop system satisfies

$$
\begin{aligned}
\left.n_{\hat{F}}^{T} \dot{x}\right|_{\hat{x}} & =n_{\hat{F}}^{T}(A \hat{x}+B g(h(\hat{x}))+a) \\
& =n_{\hat{F}}^{T}\left(A \sum_{i=1}^{k+1} \lambda_{i} z_{i}+B \sum_{i=1}^{k+1} \lambda_{i} u_{w_{i}}+a\right) \\
& =\sum_{i=1}^{k+1} \lambda_{i} n_{\hat{F}}^{T}\left(A z_{i}+B u_{w_{i}}+a\right) \leq 0 .
\end{aligned}
$$

This completes the proof of (i).

(ii) Assume that condition (11) is satisfied. Choose direction $n_{D} \in \mathbb{R}^{n}$, and for all $w \in \mathcal{V}(\mathcal{C})$ an input $u_{w} \in \mathcal{V}\left(Q_{w}\right)$ such that

$\forall v \in \mathcal{V}\left(h^{-1}(w) \cap X\right): n_{D}^{T}\left(A v+B u_{w}+a\right)>0$.

Since $h^{-1}(w) \cap X$ is a convex polytope, inequality (14) at the vertices of $h^{-1}(w) \cap X$ automatically extends to the polytope itself

$\forall x \in\left(h^{-1}(w) \cap X\right): n_{D}^{T}\left(A x+B u_{w}+a\right)>0$.

Let $u=g(y)$ be the piecewise-affine static output feedback as constructed in (9). Since $u_{w} \in Q_{w}$ for all $w \in$ $\mathcal{V}(\mathcal{C})$, the sufficiency proof in (i) guarantees that output feedback law $u=g(y)$ solves Problem II.5, i.e., all nonadmissible exit facets are blocked. Therefore, it suffices to show that all state trajectories of the closed-loop system leave $X$ in finite time. We do so by showing that in every point of the state polytope $X$ the velocity in the direction of the vector $n_{D}$ is strictly positive.

Let $\hat{x} \in X$. We define $\hat{y}=h(\hat{x})$, and denote the vertex set of the corresponding simplex $S_{\hat{y}}$ by $\mathcal{V}\left(S_{\hat{y}}\right)=\left\{w_{1}, \ldots, w_{k+1}\right\}$. Like in the proof of part (i) there exist vectors $z_{i} \in h^{-1}\left(w_{i}\right) \cap X,(i=1, \ldots, k+1)$, and numbers $\lambda_{i} \in(0,1],(i=1, \ldots, k+1)$ such that:

1) $\sum_{i=1}^{k+1} \lambda_{i}=1$;

2) $\hat{y}=h(\hat{x})=\sum_{i=1}^{k+1} \lambda_{i} w_{i}$;

3) $\hat{x}=\sum_{i=1}^{k+1} \lambda_{i} z_{i}$.

Then the vector field of the closed-loop system in $\hat{x}$ satisfies

$$
\begin{aligned}
\left.n_{D}{ }^{T} \dot{x}\right|_{\hat{x}} & =n_{D}{ }^{T}(A \hat{x}+B g(h(\hat{x}))+a) \\
& =n_{D}{ }^{T}\left(A \sum_{i=1}^{k+1} \lambda_{i} z_{i}+B \sum_{i=1}^{k+1} \lambda_{i} u_{w_{i}}+a\right) \\
& =\sum_{i=1}^{k+1} \lambda_{i} n_{D}^{T}\left(A z_{i}+B u_{w_{i}}+a\right)>0
\end{aligned}
$$

where the last inequality follows from (15) and the fact that for all $i=1, \ldots, k+1$ we have $z_{i} \in h^{-1}\left(w_{i}\right) \cap X$.

Theorem IV.1 is the main result of this paper. Part (i) describes a necessary and sufficient condition for the solvability of Problem II.5 (the facet-blocking problem) in terms of a finite number of linear inequalities (the linear inequalities that define $Q_{w}$ ) that have to be satisfied for a finite number of points $w$, namely the vertices of the chamber complex $\mathcal{C}$ of $(X, h)$. Furthermore, an explicit construction of the corresponding piecewise-affine static output feedback is given. By construction, this static output feedback is also continuous. Part (ii) only provides a sufficient condition for the solvability of Problem II.6 (the control-to-facets problem).

The construction of the feedback law in the proof of Theorem IV. 1 is based on a triangulation $\mathcal{T}$ of the chamber complex $\mathcal{C}$ of $(X, h)$ with the property that $\mathcal{V}(\mathcal{T})=\mathcal{V}(\mathcal{C})$, i.e., without introducing new vertices. However, if the conditions on the inputs at the vertices of the chamber complex $\mathcal{C}$ of $(X, h)$ are fulfilled, every triangulation that is a refinement of the chamber complex $\mathcal{C}$ will lead to a piecewise-affine controller that solves the problem. If a triangulation $\mathcal{T}$ contains a vertex $w$ that is not a vertex of the chamber complex $\mathcal{C}$ of $(X, h)$, then the corresponding input $u_{w}$ has to satisfy $u_{w} \in Q_{w}$ (in case (i)). However, the existence of a suitable input is already guaranteed by the conditions described in Theorem IV.1. Therefore, the only difference that these additional vertices cause is a change in the piecewise-affine output feedback law. This is due to the fact that the chosen triangulation is used explicitly in the construction of the feedback law.

The sufficient condition of Part (ii) may be verified in a finite number of steps in a way that is similar to the state feedback case described in [11]. For a fixed direction $n_{D}$ and a fixed vertex $w$ of $\mathcal{C}$, the condition on $u_{w}$ is

$$
\forall v \in \mathcal{V}\left(h^{-1}(w) \cap X\right): n_{D}^{T}\left(A v+B u_{w}+a\right)>0 .
$$

The input $u_{w}$ can, therefore, be chosen to be a vertex of $Q_{w}$, such as the vertex maximizing $n_{D}^{T} B u_{w}$. Hence, if $\mathcal{V}(\mathcal{C})=$ $\left\{w_{i} \mid i=1, \ldots, M\right\}$, then a solution to Part (ii) can be found by enumerating all possible tuples $\left(u_{w_{1}}, \ldots, u_{w_{M}}\right)$, with $u_{w_{i}} \in$ $\mathcal{V}\left(Q_{w_{i}}\right)$ the input corresponding to output $w_{i}$, and for each possibility, attempting to solve (16) for $n_{D}$, which is a system of linear inequalities. This yields a finite algorithm.

\section{SeVERAl Examples}

\section{A. Examples of Static Output Feedback}

We start with a simple illustration of the construction of a piecewise-affine output feedback law, that solves a given control-to-facets problem. In this example, the construction of a compatible triangulation is straightforward.

1) Example V.1: Consider the system

$$
\begin{aligned}
& \dot{x}=\left(\begin{array}{rrr}
1 & -2 & 1 \\
-1 & 1 & 0 \\
1 & 1 & -2
\end{array}\right) x+\left(\begin{array}{r}
1 \\
3 \\
-4
\end{array}\right) u+\left(\begin{array}{r}
1 \\
-2 \\
3
\end{array}\right) \\
& y=\left(\begin{array}{lll}
1 & 0 & 0 \\
0 & 1 & 0
\end{array}\right) x .
\end{aligned}
$$

on state polytope $X=[0,1]^{3}$ with input set $U=[-2,2]$. Clearly, the output polytope $Y$ is $[0,1]^{2}$. We want to solve Problem II.6 with the facets $x_{1}=1$ and $x_{2}=1$ as admissible exit facets.

Let $w_{1}=(0,0), w_{2}=(1,0), w_{3}=(1,1)$, and $w_{4}=(0,1)$. The chamber complex $\mathcal{C}$ of $(X, h)$ consists of the vertices $w_{1}$, 


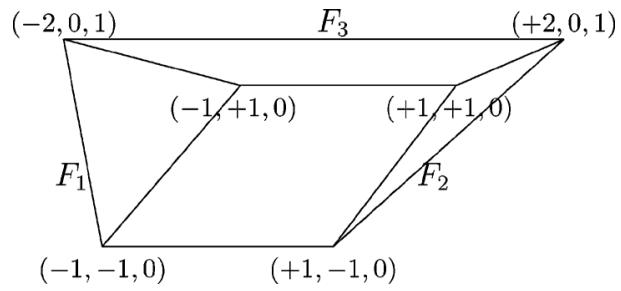

Fig. 2. Polytope $X$ defined by vertex set $\{(-2,0,1),(-1,-1,0)$, $(-1,1,0),(1,-1,0),(1,1,0),(2,0,1)\}$.

$w_{2}, w_{3}, w_{4}$, the edges joining $w_{1}$ with $w_{2}, w_{2}$ with $w_{3}, w_{3}$ with $w_{4}$, and $w_{4}$ with $w_{1}$, and finally the full chamber $[0,1]^{2}$. There are two triangulations possible, one based on the diagonal from $w_{1}$ to $w_{3}$ and the other based on the diagonal from $w_{2}$ to $w_{4}$. Both triangulations are $(X, h)$-compatible. Every vertex $w$ of $Y$ corresponds to two vertices of $X$, with $z$-coordinate 0 and 1 , respectively. According to Theorem IV.1, the input $u_{w}$ is required to be an element of the set $Q_{w}$ as defined in (10). In this example, $Q_{w}$ turns out to be nonempty for all $w \in \mathcal{V}(Y)$; straightforward computations show that the input choice $u_{w_{1}}=$ $2 / 3, u_{w_{2}}=u_{w_{3}}=u_{w_{4}}=1$ satisfies all constraints. So, a piecewise-affine output feedback law, based on the triangulation with the diagonal from $w_{2}$ to $w_{4}$, and blocking all facets except the admissible ones, is given by

$$
u=g(y)= \begin{cases}1 & \text { if } y_{1}+y_{2} \geq 1, y_{1} \leq 1, y_{2} \leq 1 \\ \frac{y_{1}+y_{2}+2}{3} & \text { if } y_{1}+y_{2}<1, y_{1} \geq 0, y_{2} \geq 0 .\end{cases}
$$

It is easily verified that in all vertices of $X$ the closed-loop vector field $\dot{x}=A x+B g(C x)+a$ has a strictly positive component in the direction of $n_{D}=(1,1,0)^{T}$. Therefore, all trajectories of the closed-loop system will leave $X$ in finite time. Hence, the output feedback law $u=g(y)$ solves Control Problem II.6.

The next example is more involved because in this case the chamber complex is nontrivial. The goal is to find a solution to a given facet-blocking problem.

2) Example V.2: Consider the polytope

$$
X=\operatorname{conv}\left\{v_{1}, v_{2}, v_{3}, v_{4}, v_{5}, v_{6}\right\}
$$

with vertices

$$
\begin{array}{ll}
v_{1}=(-1,-1,0), & v_{2}=(-1,+1,0) \\
v_{3}=(+1,-1,0), & v_{4}=(+1,+1,0) \\
v_{5}=(-2,0,+1), & v_{6}=(+2,0,+1)
\end{array}
$$

and facets

$$
\begin{array}{ll}
F_{1}=\operatorname{conv}\left\{v_{1}, v_{2}, v_{5}\right\} ; & F_{2}=\operatorname{conv}\left\{v_{3}, v_{4}, v_{6}\right\} \\
F_{3}=\operatorname{conv}\left\{v_{2}, v_{4}, v_{5}, v_{6}\right\} ; & F_{4}=\operatorname{conv}\left\{v_{1}, v_{3}, v_{5}, v_{6}\right\} \\
F_{5}=\operatorname{conv}\left\{v_{1}, v_{2}, v_{3}, v_{4}\right\} . &
\end{array}
$$

Let $h$ be the output map given by

$$
h\left(x_{1}, x_{2}, x_{3}\right)=\left(x_{1}, x_{2}\right) .
$$

The polytope $X$, the chamber complex $\mathcal{C}$ of $(X, h)$, and its triangulation are shown in Figs. 2 and 3, respectively. The chamber complex has six two-dimensional chambers, each of which is the intersection of the projection of two facets of $X$

$$
\begin{aligned}
& h\left(F_{1}\right) \cap h\left(F_{3}\right) ; \quad h\left(F_{1}\right) \cap h\left(F_{4}\right) ; \quad h\left(F_{2}\right) \cap h\left(F_{3}\right) \\
& h\left(F_{2}\right) \cap h\left(F_{4}\right) ; \quad h\left(F_{3}\right) \cap h\left(F_{5}\right) ; \quad h\left(F_{4}\right) \cap h\left(F_{5}\right) .
\end{aligned}
$$

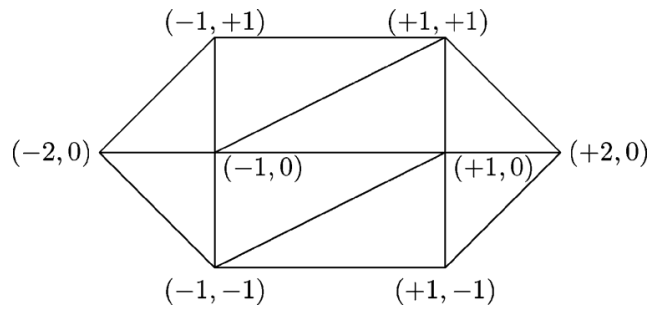

Fig. 3. Chamber complex $\mathcal{C}$ of $(X, h)$ under projection map $h\left(x_{1}, x_{2}, x_{3}\right)=$ $\left(x_{1}, x_{2}\right)$.

The vertex set of chamber complex $\mathcal{C}$ of $(X, h)$ consists of the points $w_{i}=h\left(v_{i}\right)$ for $i=1, \ldots, 6$ and the points $w_{7}=(-1,0)$ and $w_{8}=(+1,0)$. The point $w_{7}$ is the intersection of the projection of the edge joining $v_{1}$ and $v_{2}$, with the projection of the edge joining $v_{5}$ and $v_{6}$. The point $w_{8}$ is the intersection of the projection of the edge joining $v_{3}$ and $v_{4}$, with the projection of the edge joining $v_{5}$ and $v_{6}$.

Consider the facet-blocking problem with blocked facets $F_{1}$, $F_{2}$ and $F_{3}$ and corresponding outward normals

$$
n_{1}=(-1,0,-1)^{T} ; n_{2}=(+1,0,-1)^{T} ; n_{3}=(0,+1,+1)^{T} \text {. }
$$

Since the triangle $C_{1}=\operatorname{conv}\{(-2,0),(-1,0),(-1,+1)\}$ equals the intersection $h\left(F_{1}\right) \cap h\left(F_{3}\right)$, the inputs at the vertices of $C_{1}$ have to be chosen in such a way that both $F_{1}$ and $F_{3}$ are blocked at the same time. An analogous statement holds for the triangle $C_{2}=\operatorname{conv}\{(+2,0),(+1,0),(+1,+1)\}$, which is equal to $h\left(F_{2}\right) \cap h\left(F_{3}\right)$. So the inputs at the vertices of $C_{2}$ have to guarantee that both $F_{2}$ and $F_{3}$ are blocked.

Consider the affine system

$$
\dot{x}=\left(\begin{array}{rrr}
-2 & 0 & 0 \\
1 & -1 & 1 \\
0 & -1 & -1
\end{array}\right) x+\left(\begin{array}{r}
2 \\
-3 \\
1
\end{array}\right) u+\left(\begin{array}{l}
1 \\
0 \\
0
\end{array}\right) .
$$

The linear equation determining the constraint on the input $u_{i}$ at vertex $w_{i}$ of the chamber complex due to the need to block facet $F_{j}$ with normal $n_{j}$ are given from (10) as

$$
n_{j}^{T}\left(A x_{i, j}+B u_{i}+a\right) \leq 0
$$

where $x_{i, j}$ is the point on $F_{j}$ satisfying $w_{i}=h\left(x_{i, j}\right)=C x_{i, j}$.

We see that the vertex $w_{2}=(-1,+1)$ of the chamber complex is the projection of the vertex $v_{2}=(-1,+1,0)$ of the state polytope, which is a vertex of both $F_{1}$ and $F_{3}$. To block facet $F_{1}$, we require $-2-3 u_{2} \leq 0$ and to block facet $F_{3}$ we require $-3-2 u_{2} \leq 0$. The latter inequality is implied by the former, which reduces to $u_{2} \geq-2 / 3$. At the vertex $w_{3}$, we only need to block facet $F_{2}$ at vertex $v_{3}=(+1,-1,0)$, yielding the single condition $-2+u_{3} \leq 0$. At the vertex $w_{8}=(+1,0)$ there are two constraints on the input. In order to block facet $F_{2}$ at the point $x_{8,1}=(+1,0,0)$, we require $-1+u_{8} \leq 0$ and in order to block facet $F_{3}$ at the point $x_{8,2}=(+1,0,1)$ we require $1-2 u_{8} \leq 0$. By combining these two inequalities we obtain $1 / 2 \leq u_{8} \leq 1$.

Performing similar calculations at the other vertices yields

$$
\begin{aligned}
-\frac{4}{3} \leq u_{1} ; \quad-\frac{2}{3} \leq u_{2} ; \quad u_{3} \leq 2 ; \quad-\frac{1}{2} \leq u_{4} \leq 0 ; \\
-1 \leq u_{5} ; \quad 1 \leq u_{6} \leq 2 ; \quad-\frac{1}{2} \leq u_{7} ; \quad \frac{1}{2} \leq u_{8} \leq 1 .
\end{aligned}
$$




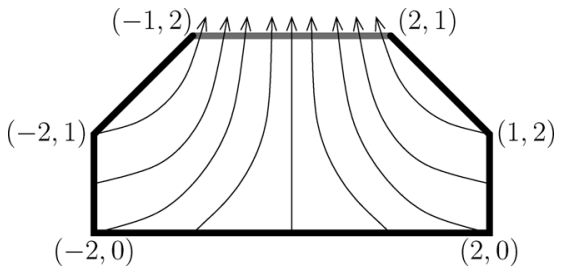

Fig. 4. Flow on polytope $X=\operatorname{conv}(\{(-2,0),(2,0),(2,1),(1,2)$, $(-1,2),(-2,1)\})$ with exit facet $[-1,1] \times\{2\}$.

Hence, the facet-blocking problem with blocked facets $F_{1}, F_{2}$ and $F_{3}$ can be solved with a piecewise-affine static output feedback with $u \in[0,1]$. Note that it is impossible to solve this facet-blocking problem with an affine static output feedback with $u \in[0,1]$.

The following example describes a system that can be controlled by an affine state feedback but not by a static output feedback.

3) Example V.3: Consider the system

$$
\dot{x}=\left(\begin{array}{cc}
0 & 0 \\
1 & 0
\end{array}\right) x+\left(\begin{array}{cc}
1 & 0 \\
0 & 1
\end{array}\right) u ; \quad y=\left(\begin{array}{ll}
0 & 1
\end{array}\right) x .
$$

on state polytope $X \subset \mathbb{R}^{2}$ given by $X=\operatorname{conv}(S)$ with vertex set $\mathcal{V}(X)=S:=\{(-2,0),(2,0),(2,1),(1,2)$, $(-1,2),(-2,1)\}$, and input polytope $U=[-6,6]^{2}$. For this system we want to solve the control-to-facet Problem II.6 with exit facet $x_{2}=2$ (see Fig. 4).

It is easily verified that this control problem is solvable by static state feedback, for example by taking

$$
u=\left(\begin{array}{ll}
-1 & 0 \\
-1 & 0
\end{array}\right) x+\left(\begin{array}{l}
0 \\
1
\end{array}\right) .
$$

However, this problem is not solvable by a piecewise-affine static output feedback. To verify this claim, we consider the input $u=g(y)$ for $y=1$. If output $y=1$, then $x_{2}=1$. So the state $x$ can be any point on the horizontal line from vertex $(-2$, 1 ) to vertex $(2,1)$. To avoid exit through the facet $x_{1}=-2$ (if $x=(-2,1)$ ), we require $\dot{x}_{1} \geq 0$, so $u_{1} \geq 0$. Similarly, to avoid exit through facet $x_{1}=2$, we need $\dot{x}_{1} \leq 0$, so $u_{1} \leq 0$. Hence, $u_{1}=0$, and in every point on the line segment from $(-2,1)$ to $(2,1)$, the first component of the closed-loop vector field is equal to 0 . Furthermore, exit through the facets $x_{1}+x_{2}=3$ or $x_{2}-x_{1}=3$ has to be avoided, which leads to the requirement $\dot{x}_{2} \leq 0$ at both $(-2,1)$ and $(2,1)$. Since $\dot{x}_{2}=x_{1}+u_{2}$ we must have $u_{2} \leq-2$ when $y=1$. This implies that in every point on the line segment from $(-2,1)$ to $(2,1)$, the second component of the closed-loop vector field is smaller than or equal to 0 . Hence, state trajectories starting with $x_{2} \leq 1$ cannot cross this line segment, and therefore, they cannot leave $X$ through the required exit facet $x_{2}=2$.

\section{B. Beyond Static Output Feedback}

Note that in Example V.3, the system is observable as a linear system, since

$$
A=\left(\begin{array}{ll}
0 & 0 \\
1 & 0
\end{array}\right) \text { and } C=\left(\begin{array}{ll}
0 & 1
\end{array}\right) \text {, so } C A=\left(\begin{array}{ll}
1 & 0
\end{array}\right) .
$$

Hence, it would be possible to control the system by a dynamic output feedback if it were possible to prevent exit of the polytope during a time interval, long enough to obtain a sufficiently accurate measurement of state $x_{1}$ to apply a robust state feed- back solution. Indeed, for the given system, we can construct a static output feedback which prevents the state from leaving the polytope, by taking

$$
u=g(y)=\left(\begin{array}{c}
0 \\
2-4 y
\end{array}\right) .
$$

Then the closed-loop system satisfies $\dot{x}_{1}=0$ and $\dot{x}_{2}=x_{1}+$ $2-4 x_{2}$. So the state vector moves along a vertical line in the direction of the fixed point $\left(x_{1}, \frac{x_{1}+2}{4}\right)$. Since $y=x_{2}$ is observed, also the value of $x_{1}$ can be determined. Since Problem II.6 is solvable by static state feedback, one may obtain an output feedback solution, by switching in finite time from the static output feedback (17) to a suitable dynamic output feedback law.

The problem of finding necessary and sufficient conditions under which Problem II.6 is solvable, using a combination of static and dynamic output feedback, requires an analysis of high-gain dynamic observers and topological robustness criteria. Partial results have been obtained by the authors, and will be reported elsewhere due to the different techniques that are required in this approach.

\section{CONCLUSION}

In this paper, we have extended the control-to-facet approach for synthesis of controllers for piecewise-affine hybrid systems to the case of systems with partial observations. The resulting control laws are continuous piecewise-affine functions of the output, with the pieces based on a triangulation of the chamber complex of the output polytope. For the facet-blocking problem, the control law can be computed by the solution of a linear programming problem; for the control-to-facet problem a sufficient condition for every solution of the closed-loop system to leave the state polytope is that there is a direction in which the flow is positive. The conditions for the existence of a solution of the facet-blocking problem are also necessary in the case that the initial state is unknown and may lie anywhere in the state polytope.

If the set of possible initial states is a subset of the state polytope, or if the state is required to leave through one of the exit facets, then dynamic output feedback may be required to solve the problem. This will be discussed in future work. Another topic for further research is to find controllers for which, for almost every initial point, the corresponding state trajectory leaves through one of the specified exit facets.

\section{APPENDIX}

In this Appendix, we state and prove some "folk theorems" on polyhedral subdivisions and triangulations that are needed in the construction of a piecewise-affine output feedback, but for which proofs are not available in the literature.

Let $P$ be a full-dimensional polytope in $\mathbb{R}^{n}$ with (closed) faces $\mathcal{F}(P)$. We denote by $P^{k}$ the $k$-skeleton of $P$, consisting of all faces of $P$ of dimension at most $k$, i.e., $P^{k}=\cup_{j=0}^{k} \mathcal{F}_{j}(P)$. Let $\pi: \mathbb{R}^{n} \rightarrow \mathbb{R}^{m}$ be a surjective affine map, and $Q:=\pi(P)$.

Definition A.1: The chamber complex $\mathcal{C}(P, \pi)$ is defined as the collection $\left\{\sigma_{(P, \pi)}(q) \mid q \in Q\right\}$, where

$$
\sigma_{(P, \pi)}(q)=\bigcap\{\pi(F) \mid F \in \mathcal{F}(P) \wedge q \in \pi(F)\}
$$

is the chamber of $q \in Q$; it is the minimal cell of the collection $\mathcal{C}(P, \pi)$ that contains $q$. 
Remark A.2: In Definition III.6 and throughout the paper, the chamber complex $\mathcal{C}(P, \pi)$ was denoted in a slightly different way as the chamber complex $\mathcal{C}$ of $(P, \pi)$. In this appendix the short notation $\mathcal{C}(P, \pi)$ is adopted. Furthermore, in the notation $\mathcal{C}(P, \pi)$ of the chamber complex and $\sigma_{(P, \pi)}(q)$ of the chamber of $q$, we omit the pair $(P, \pi)$ of polytope and affine map, if it is clear from the context to which polytope $P$ and affine map $\pi$ they refer.

It is immediate that the chamber complex $\mathcal{C}(P, \pi)$ consists of closed polytopes whose union is $\pi(P)=Q$. The goal of this appendix is to show that chamber complex $\mathcal{C}(P, \pi)$ is a polyhedral subdivision of the polytope $Q$ that is $(P, \pi)$-compatible (cf. Definition III.2).

We begin with a result that relates the faces of a polytope on the one hand and the intersection of this polytope with an affine subspace on the other.

Lemma A.3: Let $P \subset \mathbb{R}^{n}$ be a full-dimensional polytope, and $R$ an affine subspace of $\mathbb{R}^{n}$.

1) If $F \in \mathcal{F}(P)$ and $F \cap R \neq \emptyset$, then $F \cap R \in \mathcal{F}(P \cap R)$.

2) If $G \in \mathcal{F}(P \cap R)$, then there exists a unique $F \in \mathcal{F}(P)$ such that $\operatorname{relint}(G)=\operatorname{relint}(F) \cap R$.

Hence, there is a natural bijection between $\{F \in \mathcal{F}(P)$ | $\operatorname{relint}(F) \cap R \neq \emptyset\}$ and $\mathcal{F}(P \cap R)$.

Proof:

1) If $H \subset \mathbb{R}^{n}$ is a hyperplane such that $F=P \cap H$, then $F \cap R=(P \cap H) \cap R=(P \cap R) \cap H$. Since $P \cap R \subset P$ and $H$ is a supporting hyperplane of $P$ such that $H \cap R \neq \varnothing$, it follows that $H$ is also a supporting hyperplane of $P \cap R$, so $(P \cap R) \cap H$ is a face of $P \cap R$ (which is equal to $P \cap R$ if $R \subset H)$.

2) If $G=P \cap R$, then the claim is trivial. If $G \neq P \cap R$, then clearly $G \subset \partial(P \cap R)$ and since $R$ is an affine subspace, it follows that $G \subset \partial P$. Choose $x \in \operatorname{relint}(G)$. Since $x \in \partial P$, there exists a unique face $F$ of $P$ such that $x \in$ $\operatorname{relint}(F)$. By Part 1 above, $F \cap R$ is a face of $P \cap R$. Since $x \in \operatorname{relint}(F)$ and $R$ is an affine subspace, $x \in$ relint $(F \cap R)$. Since $G$ and $F \cap R$ are faces of $P \cap R$ and $x \in \operatorname{relint}(G) \cap \operatorname{relint}(F \cap R)$, we must have $G=F \cap R$.

The next result describes how the dimension of facets $F$ of $P$ and the dimension of facets $\pi(F)$ of $Q$ are related to the boundary of $\pi(F)$ and the image of the boundary of $F$.

Lemma A.4: Let $P \subset \mathbb{R}^{n}$ be a full-dimensional polytope, $\pi: \mathbb{R}^{n} \rightarrow \mathbb{R}^{m}$ be surjective and $Q=\pi(P)$. Then:

1) if $F \in \mathcal{F}(P)$ and $\operatorname{dim}(\pi(F))=\operatorname{dim}(F)$, then $\partial \pi(F)=$ $\pi(\partial F)$;

2) if $F \in \mathcal{F}(P)$ and $\operatorname{dim}(\pi(F))<\operatorname{dim}(F)$, then $\pi(F)=$ $\pi(\partial F)$

3) if $G \in \mathcal{F}(Q)$, then there exists $F \in \mathcal{F}(P)$ such that $G=$ $\pi(F)$.

Proof:

1) If $\operatorname{dim}(\pi(F))=\operatorname{dim}(F)$, then $\left.\pi\right|_{F}$ is injective. So $\left.\pi\right|_{F}$ is a bijective continuous mapping from $F$ to $\pi(F)$. Therefore, we must have $\partial \pi(F)=\pi(\partial F)$.

2) Since the inclusion $\pi(\partial F) \subset \pi(F)$ is trivial, we only have to show that $\pi(F) \subset \pi(\partial F)$. Let $y \in \pi(F)$. Then there exists $x_{1} \in F$ such that $y=\pi\left(x_{1}\right)$. If $x_{1} \in \partial F$, then $y \in \pi(\partial F)$, and we are done. If $x_{1} \notin \partial F$, then there exists an $x_{2} \in F$, such that $\pi\left(x_{2}\right)=y$ and $x_{2} \neq x_{1}$, because $x_{1} \in \operatorname{relint}(F)$ and the affine mapping $\left.\pi\right|_{F}$ is not injective since $\operatorname{dim}(\pi(F))<\operatorname{dim}(F)$.

Let $\ell$ be the straight line through $x_{1}$ and $x_{2}$. Then for all $x \in \ell$ we have $\pi(x)=y$, and the line $\ell$ is contained in the supporting hyperplane of $F$. So $\ell$ intersects the boundary of $F$. Choose $x_{3} \in \ell \cap \partial F$. Then $\pi\left(x_{3}\right)=y$, and therefore, $y \in \pi(\partial F)$.

3) Let $H$ be a hyperplane in $\mathbb{R}^{m}$ such that $Q \cap H=G$. Then $\pi^{-1}(H)$ is a supporting hyperplane of $P$, and we can take $F=P \cap \pi^{-1}(H)$.

The following lemma is trivial.

Lemma A.5: If $y \in \sigma_{(P, \pi)}(x)$, then $\sigma_{(P, \pi)}(y) \subset \sigma_{(P, \pi)}(x)$.

Proof: Immediate from $\{F \in \mathcal{F}(P) \mid y \in \pi(F)\} \supset\{F \in$ $\mathcal{F}(P) \mid x \in \pi(F)\}$.

We now briefly recall some simple facts about sets and functions. For any function $f, f(A \cap B) \subset f(A) \cap f(B)$ with equality if $f$ is injective, $f\left(f^{-1}(B)\right) \subset B$ with equality if $f$ is surjective, and $f\left(A \cap f^{-1}(B)\right)=f(A) \cap B$.

Lemma A.6: Let $S$ be an affine subspace of $\mathbb{R}^{m}$, and set $R=\pi^{-1}(S)$. Suppose $x, y \in \pi(P) \cap S$. Then $\sigma_{(P, \pi)}(x)=$ $\sigma_{(P, \pi)}(y)$ if, and only if, $\sigma_{\left(P \cap R,\left.\pi\right|_{R}\right)}(x)=\sigma_{\left(P \cap R,\left.\pi\right|_{R}\right)}(y)$.

Proof: Suppose $F \in \mathcal{F}(P)$ and $x \in \pi(F)$. Then by Lemma A.4(2) there exists a face $G \subset F$ such that $\pi$ is injective on $G$ and $x \in \pi(\operatorname{relint}(G))=\operatorname{relint}(\pi(G))$. Since $\pi$ is injective on $\operatorname{relint}(G)$ and $x \in S$, we have

$$
\begin{aligned}
\pi(\operatorname{relint}(G) \cap R) & =\pi(\operatorname{relint}(G)) \cap \pi(R) \\
& =\operatorname{rel} \operatorname{int}(\pi(G)) \cap S \ni x
\end{aligned}
$$

so $\operatorname{relint}(G) \cap R \neq \emptyset$. Hence

$\sigma_{(P, \pi)}(x)=\bigcap\{\pi(F) \mid F \in \mathcal{F}(P)$

$$
\text { s.t. } \operatorname{relint}(F) \cap R \neq \emptyset \text { and } x \in \pi(F)\}
$$

and a similar consideration holds for $\sigma_{(P, \pi)}(y)$.

By Lemma A.3, $\{F \cap R \mid F \in \mathcal{F}(P)$ and $\operatorname{relint}(F) \cap R \neq$ $\emptyset\}=\mathcal{F}(P \cap R)$, so

$\{G \in \mathcal{F}(P \cap R) \mid x \in \pi(G)\}$

$=\{F \cap R \mid F \in \mathcal{F}(P)$ s.t. $\operatorname{relint}(F) \cap R \neq \emptyset$ and $x \in \pi(F)\}$.

If $\sigma_{(P, \pi)}(x)=\sigma_{(P, \pi)}(y)$, then $\{F \in \mathcal{F}(P) \mid x \in \pi(F)\}=$ $\{F \in \mathcal{F}(P) \mid y \in \pi(F)\}$, and from the above considerations we have $\{G \in \mathcal{F}(P \cap R) \mid x \in \pi(G)\}=\{G \in \mathcal{F}(P \cap R) \mid$ $y \in \pi(G)\}$.

Conversely, if $\sigma_{\left(P \cap R,\left.\pi\right|_{R}\right)}(x)=\sigma_{\left(P \cap R,\left.\pi\right|_{R}\right)}(y)$, we have $\{G \in \mathcal{F}(P \cap R) \mid x \in \pi(G)\}=\{G \in \mathcal{F}(P \cap R) \mid y \in \pi(G)\}$, and can deduce

$$
\begin{aligned}
& \{F \in \mathcal{F}(P) \mid \operatorname{relint}(F) \cap R \neq \emptyset \text { and } x \in \pi(F)\} \\
& \quad=\{F \in \mathcal{F}(P) \mid \operatorname{relint}(F) \cap R \neq \emptyset \text { and } y \in \pi(F)\} .
\end{aligned}
$$

Hence

$$
\begin{aligned}
\{F \in \mathcal{F}(P) \mid F \cap R \neq \emptyset \text { and } x \in \pi(F)\} \\
\\
=\{F \in \mathcal{F}(P) \mid F \cap R \neq \emptyset \text { and } y \in \pi(F)\} .
\end{aligned}
$$


Since $x, y \in S=\pi(R)$, this implies $\{F \in \mathcal{F}(P) \mid x \in$ $\pi(F)\}=\{F \in \mathcal{F}(P) \mid y \in \pi(F)\}$ and so $\sigma_{(P, \pi)}(x)=$ $\sigma_{(P, \pi)}(y)$.

The previous lemma often allows us to reduce statements to either that of full-dimensional cells, or to one-dimensional images. The following crucial lemma relates chambers of points lying on a line segment.

Lemma A.7: Let $x, y \in Q$ with $\sigma(x) \subset \sigma(y)$. Then $\sigma(z)=$ $\sigma(y)$ for all $z$ on the line segment between $x$ and $y$ with $z \neq x$.

Proof: Clearly, if $x, y \in \pi(F)$ and $z$ lies between $x$ and $y$, then $z \in \pi(F)$. By Lemma A.6, by restricting to the line between $\sigma(x)$ and $\sigma(y)$, we can assume $\operatorname{dim}(Q)=1$.

Suppose there exists a face $F$ of $P$ such that there exists a point between $x$ and $y$ in $\pi(F)$ but $x, y \notin \pi(F)$. Since if $\operatorname{dim}(F)>1$ we have $\pi(F)=\pi(\partial F)$ by Lemma A.4(2), we can find a possibly different $F$ with $\operatorname{dim}(F)=1$. Let $w$ be the closest point of $\pi(F)$ to $\pi(x)$. Then $w \in \pi(\partial F)$ by Lemma A.4(1), and since $\operatorname{dim}(F)=1$, there exists vertex $v$ of $P$ such that $\pi(v)=w$. Since $v$ is a vertex of $P$, we have that for all $u \in P$, there exist $\lambda_{i} \geq 0$ such that $u=v+\sum_{i=0}^{k} \lambda_{i} e_{i}$ where $e_{i}$ are the directions of the edges (1-faces) of $P$ starting at $v$. Taking $u \in \pi^{-1}(x)$, we see that there is an edge $E$ starting at $v$ with tangent vector $e$ such that $\pi(e)$ points in the direction of $x$; in particular, $y \notin \pi(E)$. Since the vertex at the end of $E$ does not project between $x$ and $w$, we have $x \in \pi(E)$. Hence, $x$ and $y$ do not lie in the interior of the same chamber of $Q$, a contradiction.

Corollary A.8: Suppose $y \in \sigma(x)$. Then $\sigma(y)=\sigma(x)$ or $\sigma(y) \subset \partial \sigma(x)$. In particular, if $y \in \operatorname{relint} \sigma(x)$, then $\sigma(y)=$ $\sigma(x)$.

Proof: If $\sigma(y) \not \subset \partial \sigma(x)$, there exists $z \in \sigma(y) \cap$ relint $\sigma(x)$. Then there exists $w \in \sigma(x)$ such that $z$ lies in the relative interior of the line segment joining $w$ and $x$, so $\sigma(z)=\sigma(x)$ by Lemma A.7. However, since $z \in \sigma(y)$ and $y \in \sigma(z)$ we have $\sigma(z) \subset \sigma(y) \subset \sigma(x)$ by Lemma A.5. Hence, $\sigma(x)=\sigma(y)$.

The following result is converse to Lemma A.7

Corollary A.9: Suppose $y \in \partial \sigma(x)$. Then $\sigma(y) \subset \partial \sigma(x)$.

Proof: By Lemma A.6, it suffices to consider the case $\operatorname{dim}(\sigma(x))=\operatorname{dim}(Q)=m$. By Lemma A.4, there is at least one face $F$ of $P$ of dimension $m-1$ such that $y \in \pi(F)$. Hence, $\operatorname{dim}(\sigma(y))<\operatorname{dim}(\sigma(x))$, so $\sigma(y) \neq \sigma(x)$. Hence, by Corollary A.8, $\sigma(y) \subset \partial \sigma(x)$.

The following lemma is also crucial; it shows that any chamber which is a subset of another is actually a face of the other chamber.

Lemma A.10: Let $x, y \in P$ and $\sigma(y) \subset \sigma(x)$. Then $\sigma(y)$ is a face of $\sigma(x)$.

Proof: By Lemma A.6, it suffices to consider the case $\operatorname{dim}(\sigma(x))=\operatorname{dim}(Q)=m$. By Corollary A.9 if $\sigma(y) \subsetneq \sigma(x)$, then $\sigma(y) \subset \partial \sigma(x)$. Further, it clearly suffices to consider the case $\operatorname{dim}(\sigma(y))=\operatorname{dim}(Q)-1$, since otherwise we could restrict to a proper face of $\sigma(x)$. Hence, $\sigma(y)$ is a polyhedral subset of $\partial \sigma(x)$.

Suppose $\sigma(y)$ is not a facet of $\sigma(x)$. Then there is a facet of $\sigma(y)$ which intersects the relative interior of a facet of $\sigma(x)$. We can, therefore, choose $z \in \sigma(y)$ such that $\operatorname{dim}(\sigma(z))=m-2$ and $z$ lies in the relative interior of a facet of $\sigma(x)$. We need to consider two cases, either there is an $(m-2)$-dimensional face $F$ of $P$ such that $z \in \pi(F)$, or there is no such face, but there are two $(m-1)$-dimensional faces $F_{1}, F_{2}$ of $P$ such that $\operatorname{dim}\left(\pi\left(F_{1}\right) \cap \pi\left(F_{2}\right)\right)=m-2$ and $z \in \pi\left(\operatorname{relint}\left(F_{1}\right)\right) \cap$ $\pi\left(\operatorname{relint}\left(F_{2}\right)\right)$.

Consider the first possibility, that there is an $(m-2)$-dimensional face $F$ of $P$ such that $z \in \pi(F)$. Let $p$ be the (unique) point in $F$ with $\pi(p)=z$. Choose a vector $v$ at $p$ parallel to $\partial P$ such that $\pi(v)$ points from $z$ inside $\sigma(y)$. Then $F$ and $v$ span an $(m-1)$-dimensional face $G$ of $P$ such that $\pi(G)$ intersects the interior of $\sigma(x)$. Hence, there is a point $w \in \operatorname{relint}(\sigma(x))$ such that $\operatorname{dim}(\sigma(w))<\operatorname{dim}(\sigma(x))$, which contradicts Corollary A.8.

Consider the second possibility. Then it cannot be the case that both $F_{1}$ and $F_{2}$ map under $\pi$ into the $m$-1-dimensional subspace spanned by $\sigma(y)$, for then their intersection would have dimension $m-1$. Without loss of generality, suppose that $F_{1}$ does not map into the subspace spanned by $\sigma(y)$. Then $\pi\left(F_{1}\right)$ intersects the relative interior of $\sigma(x)$, again a contradiction.

Theorem A.11: Let $P \subset \mathbb{R}^{n}$ be a full-dimensional polytope, $\pi: \mathbb{R}^{n} \rightarrow \mathbb{R}^{m}$ be surjective, and $Q=\pi(P)$. Then the chamber complex $\mathcal{C}(P, \pi)$ is a polyhedral subdivision of $Q$.

Proof: Since every chamber $\sigma(x)$ is the intersection of convex sets, it is itself convex. If $y \in \sigma(x)$, then $\sigma(y) \subset \sigma(x)$. By Lemma A.7, for any $x$, the set $\{y \mid \sigma(y)=\sigma(x)\}$ is convex, and is the relative interior of $\sigma(x)$. In particular, if $\sigma(y) \subsetneq \sigma(x)$, then $y \in \partial \sigma(x)$.

If $F$ is a face of $\sigma(x)$ and $y \in \operatorname{relint}(F)$, then $\sigma(y) \subset F$ by Corollary A.9, and $\sigma(y) \supset F$ by Lemma A.10. Hence, all the faces of a chamber are also chambers.

If $\sigma(x)$ and $\sigma(y)$ are chambers, then $\sigma(x) \cap \sigma(y)$ is either empty, or a polytope. Choose $z$ in the relative interior of this intersection. Then $\sigma(z)$ is a face of both $\sigma(x)$ and $\sigma(y)$ by Lemma A.10. Hence, $\sigma(z)=\sigma(x) \cap \sigma(y)$.

Recall from Definition III.2 that a polytopic subdivision $\mathcal{Q}$ of $Q$ is $\pi$-compatible if for every full-dimensional cell $G$ of $\mathcal{Q}$, we have $\pi\left(\mathcal{V}\left(\pi^{-1}(G) \cap P\right)\right) \subset \mathcal{V}(G)$.

Theorem A.12: Let $P \in \mathbb{R}^{n}$ be a full-dimensional polytope, $\pi: \mathbb{R}^{n} \rightarrow \mathbb{R}^{m}$ be a surjective linear map, and $Q=\pi(P)$. Let $\mathcal{C}$ be the chamber complex of $Q$ induced by $\pi$. Then a polyhedral subdivision $\mathcal{Q}$ of $Q$ is $\pi$-compatible if $\mathcal{Q}$ is a refinement of $\mathcal{C}$. If $\mathcal{Q}$ is a simplicial subdivision (triangulation), then $\mathcal{Q}$ is $\pi$-compatible only if it is a refinement of $\mathcal{C}$.

Proof: Suppose $\mathcal{Q}$ is a polyhedral subdivision of $Q$ refining $\mathcal{C}$, and let $G$ be a cell of $\mathcal{Q}$. Then the chamber complex of $P \cap$ $\pi^{-1}(G)$ under $\pi$ has a single full-dimensional cell, namely $G$. Since by Lemma A.4 $(1,2)$ the $k$-skeleton of any polytope maps into the $k$-skeleton of the chamber complex, it follows by taking $k=0$ that $\mathcal{Q}$ is compatible.

Conversely, suppose that $\mathcal{Q}$ is a compatible triangulation. Let $G$ be an $m$-dimensional cell of $\mathcal{Q}$ and $F$ a face of $P$ such that $\pi(F)$ intersects the interior of $G$. By Lemma A.4, we can, without loss of generality, assume that $\pi$ is injective on $F$, and so $\pi\left(\mathcal{V}\left(\pi^{-1}(G) \cap F\right)\right)=\mathcal{V}\left(\pi\left(\pi^{-1}(G) \cap F\right)\right)=\mathcal{V}(G \cap \pi(F))$. Since $\mathcal{Q}$ is $\pi$-compatible with $P$, we have $\pi\left(\mathcal{V}\left(\pi^{-1}(G) \cap P\right) \subset\right.$ $\mathcal{V}(G)$ by definition. Since $F$ is a face of $P, \mathcal{V}\left(\pi^{-1}(G) \cap F\right)=$ $\mathcal{V}\left(\pi^{-1}(G) \cap P\right) \cap F$. 
Hence

$$
\begin{aligned}
& \mathcal{V}(G \cap \pi(F)) \\
& =\pi\left(\mathcal{V}\left(\pi^{-1}(G) \cap F\right)\right)=\pi\left(\mathcal{V}\left(\pi^{-1}(G) \cap P\right) \cap F\right) \\
& =\pi\left(\mathcal{V}\left(\pi^{-1}(G)\right) \cap P\right) \cap \pi(F) \subset \mathcal{V}(G) \cap \pi(F)
\end{aligned}
$$

Thus, $\mathcal{V}(G \cap \pi(F)) \subset \mathcal{V}(G) \cap \pi(F) \subset \mathcal{V}(G)$. Since $G$ is a simplex and $\pi(F)$ is a polytope, this implies $G \subset \pi(F)$. Since $G \subset \pi(F)$ whenever $\operatorname{int}(G) \cap \pi(F) \neq \emptyset$, we have $G \subset \sigma(x)$ for any $x \in \operatorname{int}(G)$. Hence, $\mathcal{Q}$ refines $\mathcal{C}$.

\section{ACKNOWLEDGMENT}

Prof. J. Rambau is gratefully acknowledged for his advice on triangulations and the chamber complex. The authors thank the anonymous reviewers for their useful comments.

\section{REFERENCES}

[1] A. Balluchi, F. Di Natale, A. L. Sangiovanni-Vincentelli, and J. H. van Schuppen, "Synthesis for idle speed control of an automotive engine," in Hybrid Systems: Computation and Control, Proc. 7th International Workshop HSCC, R. Alur and G. J. Pappas, Eds. Berlin, Germany: Springer-Verlag, 2004, vol. 2993, pp. 80-94, Lecture Notes Comput. Sci.

[2] C. Belta and L. C. G. J. M. Habets, "Controlling a class of nonlinear systems on rectangles," IEEE Trans. Autom. Control, vol. 51, no. 11, pp. 1749-1759, Nov. 2006.

[3] A. Bemporad and M. Morari, "Control of systems integrating logic, dynamics, and constraints," Automatica, vol. 35, pp. 407-427, 1999.

[4] A. Bemporad, G. Ferrari-Trecate, and M. Morari, "Observability and controllability of piecewise affine and hybrid systems," IEEE Trans. Autom. Control, vol. 45, no. 10, pp. 1864-1876, Oct. 2000.

[5] M. E. Broucke, "Reach control on simplices by continuous state feedback," SIAM J. Control Optim., vol. 48, pp. 3482-3500, 2010.

[6] P. Collins, L. Habets, A. Kuut, M. Nool, M. Petreczky, and J. H. van Schuppen, "ConPAHS - A software package for control of piecewiseaffine hybrid systems," in Proc. IEEE Conf. Computer Aided Control Systems Design, Munich, Germany, 2006, pp. 76-81.

[7] J. D. Grunnet, J. A. Larsen, T. Bak, and R. Wisniewski, K. Fletcher, Ed., "A piecewise affine hybrid systems approach to fault tolerant satellite formation control," in Proc. 3rd Int. Symp. Formation Flying, Missions and Technologies, Noordwijk, The Netherlands, 2008.

[8] J. D. Grunnet, T. Bak, J. D. Bendtsen, and F. Ankersen, "PAHSCTRL - A control synthesis toolboox for piecewise-affine hybrid systems," presented at the 10th Eur. Control Conf., Budapest, Hungary, 2009.

[9] L. C. G. J. M. Habets and J. H. van Schuppen, "A control problem for affine dynamical systems on a full-dimensional polytope," Automatica, vol. 40, pp. 21-35, 2004.

[10] L. C. G. J. M. Habets and J. H. van Schuppen, "Control to facet problems for affine systems on simplices and polytopes - with application to control of hybrid systems," in Proc. 44th IEEE Conf. 2005, Seville, Spain, Dec. 2005, pp. 4175-4180.

[11] L. C. G. J. M. Habets, P. J. Collins, and J. H. van Schuppen, "Reachability and control synthesis for piecewise-affine hybrid systems on simplices," IEEE Trans. Autom. Control, vol. 51, no. 6, pp. 938-948, Jun. 2006.

[12] C. W. Lee, "Subdivisions and triangulations of polytopes," in Handbook of Discrete and Computational Geometry. Boca Raton, FL: CRC, 1997, CRC Press Ser. Discrete Math. Appl., pp. 271-290.

[13] Z. Lin and M. E. Broucke, "Resolving control to facet problems for affine hypersurface systems on simplices," in Proc. 45th IEEE Conf. Decision and Control, San Diego, CA, 2006, pp. 2625-2630.

[14] Z. Lin and M. E. Broucke, "On a reachability problem for affine hypersurface systems on polytopes," Automatica, vol. 47, pp. 769-775, 2011.

[15] J. A. De Loera, J. Rambau, and F. Santos, Triangulations: Structures for Algorithms and Applications. Berlin, Germany: Springer-Verlag, 2010, vol. 25, Algorithms and Computation in Mathematics.

[16] J. Rambau, "Polyhedral Subdivisions and Projections of Polytopes," Ph.D. thesis, Tech. Univ. Berlin, Berlin, Germany, 1996.

[17] J. Rambau and G. M. Ziegler, "Projections of polytopes and the generalized Baues conjecture," Discrete Comupt. Geom., vol. 16, pp. 215-237, 1996.
[18] B. Roszak and M. E. Broucke, "Necessary and sufficient conditions for reachability on a simplex," Automatica, vol. 42, pp. 1913-1918, 2006.

[19] B. Roszak and M. E. Broucke, "Reachability of a set of facets for linear affine systems with $n-1$ inputs," IEEE Trans. Autom. Control, vol. 52, no. 2, pp. 359-364, Feb. 2007.

[20] E. D. Sontag, "Nonlinear regulation: The piecewise linear approach," IEEE Trans. Autom. Control, vol. AC-26, no. 2, pp. 346-358, Apr. 1981.

[21] E. D. Sontag, "Interconnected automata and linear systems: A theoretical framework in discrete-time," in Hybrid Systems III: Verification and Control, R. Alur, T. A. Henzinger, and E. D. Sontag, Eds. Berlin, Germany: Springer-Verlag, 1996, vol. 1066, pp. 436-448, Lecture Notes Comput. Sci.

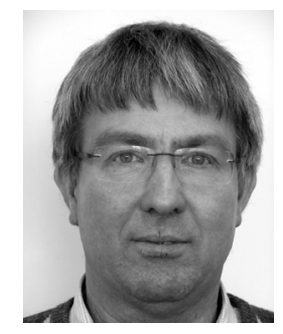

Luc C. G. J. M. Habets received the M.Sc. degree in applied mathematics and the Ph.D. degree from the Eindhoven University of Technology, Eindhoven, The Netherlands, in 1989 and 1994, respectively.

From 1994 until 1997, he was affiliated with the Institute for Dynamical Systems at Bremen University, Germany. He returned to Eindhoven in 1997 to become a Lecturer with the Department of Mathematics and Computer Science. Since 2000, he has also been part-time affiliated as Researcher with the Centrum Wiskunde \& Informatica (CWI), Amsterdam, The Netherlands. His main research interests include hybrid systems, time-delay systems, behavioral theory, and algebraic and computational aspects in systems and control.

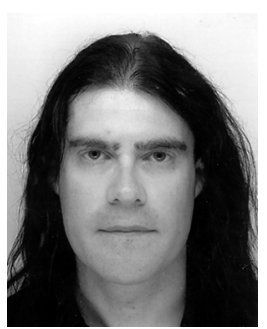

Pieter J. Collins received the B.A. Hons (1 st Class) degree in mathematics in 1994 and the Distinction in Part III of the Mathematics Tripos in 1995 from Cambridge University, Cambridge, U.K. He received the $\mathrm{Ph} . D$. degree in applied mathematics in 1999 from the University of California at Berkeley under the supervision of Prof. M. Hirsch for a thesis entitled "Dynamics of Surface Maps with Homoclinic and Heteroclinic Tangles".

From 2000-2002, he was with the University of Liverpool, Liverpool, U.K., supported by a Leverhulme Special Research Fellowship, and from 2003 to 2010, with the Centrum Wiskunde \& Informatica (CWI), Amsterdam, The Netherlands, from 2005-2010 supported by a Vidi grant from the Nederlandse Organisatie voor Wetenschappelijke Onderzoek (Netherlands Organisation for Scientific Research, NWO) for the project "Computational Topology for Systems and Control”. Since 2011, he has been an Assistant Professor at the Department of Knowledge Engineering, Maastricht University, Maastricht, The Netherlands. His main research interests are in chaotic dynamical systems, differential inclusions, reachability analysis and verification of hybrid systems, and computability theory for continuous mathematics, and rigorous numerical methods.

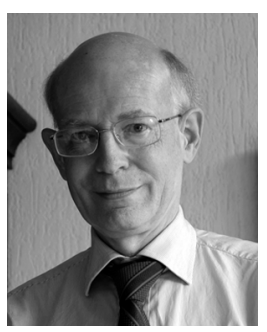

Jan H. van Schuppen is a CWI Fellow with the research institute Centrum Wiskunde \& Informatica (CWI), Amsterdam, The Netherlands, and a Full Professor (part time) with the Department of Mathematics of the Delft University of Technology, Delft, The Netherlands. His research interests include control of hybrid systems and of discrete-event systems, stochastic control, realization, and system identification. In applied research, his interests include control of distributed/decentralized systems, engineering problems of control of motorway traffic, of communication networks, and control and system theory for the life sciences. He regularly teaches courses for Ph.D. and master students. Finally, he was the coordinator of the $\mathrm{C} 4 \mathrm{C}$ Project (CON4COORD, Grant agreement INFSO-ICT-223844) and was involved in seven more of such projects.

Dr. van Schuppen is Co-Editor of the journal Mathematics of Control, Signals, and Systems, was Associate Editor-at-Large of the IEEE TRANSACTIONS AUTOMATIC CONTROL, and was Department Editor of the journal Discrete Event Dynamic Systems. 